\title{
All Compact Hausdorff Lambda Models are Degenerate
}

\author{
K. H. Hofmann* \\ Fachbereich Mathematik \\ Schloßgartenstraße 7 \\ D-64289 Darmstadt \\ Germany \\ and \\ M. W. Mislove* \\ Department of Mathematics \\ Tulane University \\ New Orleans, LA 70118 \\ Fundamenta Informaticae, to appear
}

\begin{abstract}
The first mathematical model of the untyped lambda calculus was discovered by DANA SCOTT in the category of algebraic lattices and Scott continuous maps. The question then arises as to which other cartesian closed categories contain a model of the calculus. In this paper we show that any compact Hausdorff model of the calculus must satisfy the property that the semantic map from the calculus to the model is constant. In particular, any compact reflexive object in the category of Hausdorff $k$-spaces gives rise to a degenerate model of the calculus. We also explore the relationship of the results we derive to the notions of a combinatory model and of an environment model of the calculus.
\end{abstract}

Dedication. Dedicated to Dana S. ScotT on the occasion of his sixty-first birthday on October 11, 1993 as an expression of our admiration and appreciation for the continuous inspiration coming from him to topology and algebra. He makes us think about theory while keeping applications in mind.

\section{Introduction}

The lambda calculus of $\mathrm{CHURCH}$ and CURRY long has been an object of study by researchers in theoretical computer science. The reason is that the untyped lambda calculus is very much like a prototypical programming language without assignment.

* The first author thanks Deutsche Forschungsgemeinschaft and the second the Office of Naval Research for their support. E-mail addresses: first author: hofmann@mathematik.th-darmstadt.de, and second author: mwm@tulmath.math.tulane.edu. 
As such, this calculus provides an attractive basis for building models for programming languages. But the question arises as to the existence of mathematical models of the calculus. By such a model, we mean one where the terms of the calculus can be interpreted as functions, and where the application and abstraction operators have the obvious natural meaning. It was only in the late 1960's that DANA SCOTT provided the first mathematical model of the calculus. This result was an offshoot of his attempt to provide mathematical models for programming languages other than the lambda calculus, a calculus whose use he viewed as formal and unmotivated (cf. [2], p. vii). His first model, the so-called $D_{\infty}$ model ([5]), was found in the category of algebraic lattices and Scott continuous maps. His investigations led to the founding of domain theory - objects which are generalizations of algebraic lattices within the realm of partially ordered sets - and a veritable plethora of models for the calculus has been found in categories of domains.

An obvious question then is whether models for the calculus can be found in other categories. Following MEYER (cf. [6]), by a functional domain, we mean an object $X$ within a cartesian closed category $\mathcal{C}$ with internal hom functor $\Rightarrow$ which satisfies the property that $X \Rightarrow X$ is a retract of $X$ in $\mathcal{C}$. ${ }^{1}$ The model $D_{\infty}$ is an example of such an object; indeed, $D_{\infty} \simeq\left(D_{\infty} \Rightarrow D_{\infty}\right)$. So, one would like to know whether there exist such objects in other cartesian closed categories.

The place to start such an investigation is clearly $\mathcal{S E T}$, the category of sets and functions. Here, the internal hom functor is the family of all functions, and then Cantor's Theorem on cardinalities implies there can be no nondegenerate functional domain in the category. Other categories of interest are $\mathcal{P O S}$, the category of posets and monotone maps, and the category $\mathcal{C U}$ of complete ultrametric spaces and nonexpansive maps. Arguments exist to show that neither of these categories admits a nondegenerate functional domain.

The next category on the list of candidates is the category $\mathcal{K}$ of Hausdorff $k$-spaces and continuous maps. For this category, the question remains open whether there is a nondegenerate functional domain. Indeed, the purpose of this paper is to eliminate an obvious subclass of objects as candidates for nondegenerate functional domains - the compact spaces. The precise aspects, if one wants to present them in sufficient generality, are technical and sometimes subtle. We introduce concepts which relate to the issue of "compact lambda models": the first one is that of a functional algebra in (2.1), which is a certain topological algebraic structure. All subsequent results rest on the fact that Hausdorff compact functional algebras must be singleton (2.7). The proof relies on results from the theory of compact semigroups and compact semigroup actions. We define a functional domain and its variants in considerable generality in (3.1). The ones we introduced above we shall call special.

In the third and fourth sections we also take the time to discuss in some detail the precise effect of our results on models of the untyped lambda calculus in cartesian closed topological categories. This discourse is motivated by MEYER's ideas [6]. In particular, we show that an environment model based on a compact special functional domain in the category of $k$-spaces must be trivial in the sense that its semantic map is constant (3.11). In the fourth section we discuss briefly the concept of combinatory models in concrete cartesian closed categories and the effects of our degeneracy theorem on the degeneracy of compact extensional combinatory models in, say the category of $k$-spaces $(4.5,4.6)$.

The first two sections are point-set topological; no category theory is used there. In the subsequent sections, however, we proceed in sufficient generality so that most of the results apply to arbitrary concrete cartesian closed categories with

1 Actually, this definition is somewhat more restrictive than MEYER's, focusing on the case when the family of self-maps is all of $X \Rightarrow X$. 
functions spaces. This requires some background on cartesian closed categories. Therefore, basic aspects of cartesian closed categories are collected in an Appendix for easy reference. As soon as the results of the first point-set topological sections and the category-based arguments merge, we work in what we call standard topological categories, i.e., cartesian closed categories based in the category of topological spaces.

We are indebted to SAMSOn ABRAMSKy for bringing the problem of models of the lambda calculus within $\mathcal{K}$ to our attention.

\section{Preliminaries on Effective Topological Monoid Actions}

A topological semigroup $T$ is a Hausdorff topological space together with a continuous associative multiplication $(s, t) \mapsto s t: T \times T \rightarrow T$. Our core results will involve compact Hausdorff topological semigroups.

Definition 1.1. Suppose that $(T, e)$ is a topological semigroup with an idempotent $\epsilon$. We say that $(T, e)$ acts e-effectively on a topological space $X$ if there is a continuous $\operatorname{map}(t, x) \mapsto t \cdot x: T \times X \rightarrow X$ satisfying

$$
\begin{gathered}
\left(\forall t_{1}, t_{2} \in T, x \in X\right) t_{1} t_{2} \cdot x=t_{1} \cdot\left(t_{2} \cdot x\right), \\
(\forall x \in X) e \cdot x=x .
\end{gathered}
$$

We say that the action is e-effective if

$$
\left(\forall t_{1}, t_{2} \in T\right)\left((\forall x \in X) t_{1} \cdot x=t_{2} \cdot x \Rightarrow e t_{1}=e t_{2} \cdot\right)
$$

If $e$ is an identity, then we recover the standard concept of an effective action. We shall refer to $e$ as being central if et $=$ te for all $t \in T$.

Lemma 1.2. Suppose that $(T, e)$ acts e-effectively on $X$. Then

$$
(\forall t \in T) \text { et }=\text { ete. }
$$

Moreover, we have the following conclusions:

(i) The morphism $p: T \rightarrow T, p(t)=$ et is a homomorphic retraction onto a subsemigroup

$$
S \stackrel{\text { def }}{=} e T \text {. }
$$

(ii) $S$ is a left ideal and, if e is central, an ideal of $T$.

(iii) $S$ is a monoid with identity $e$ acting effectively on $X$.

(iv) $(\forall t \in T, x \in X) t \cdot x=p(t) \cdot x$.

Proof. In order to prove (4) we compute $(t e) \cdot x=t \cdot(e \cdot x)=t \cdot x$. So by (3) we have $e t e=e t$. Now (i) is elementary semigroup theory, (ii) is trivial from $S=e T$ (which equals $T e$ if $e$ is central). (iii) is straightforward and in order to see (iv), we compute $t \cdot x=e \cdot(t \cdot x)=(e t) \cdot x=p(t) \cdot x$.

Definition 1.3. We shall call $S=e T$ the structure monoid of the action of $(T, e)$ on $X$.

The following remarks shed some extra light on ways in which a structure monoid might be embedded in the full semigroup. If $p: T \rightarrow T$ is a retraction of set onto a subset $S$ which is a semigroup, then the definition $t_{1} t_{2}=p\left(t_{1}\right) p\left(t_{2}\right)$ makes $T$ into a semigroup called the inflation of $S$ via $p$. If $e$ is an identity of $S$, then $e$ is a central idempotent of $T$. 
Remark 1.4. Suppose that $T$ is a semigroup with an idempotent $e$ satisfying (4). Set $S=e T$. Then the following two statements are equivalent:

(a) $T T \subseteq S$.

(b) $T$ is an inflation of $S$ via $p=(t \mapsto e t)$.

Proof. $\quad(\mathrm{b}) \Rightarrow(\mathrm{a})$ : We note $t_{1} t_{2}=p\left(t_{1}\right) p\left(t_{2}\right)=$ et $_{1} e t_{2}=$ et $_{1} t_{2}$ by $(4)$, and the last element is in $e T=S$.

$(\mathrm{a}) \Rightarrow(\mathrm{b})$ : Let $t_{1}, t_{2} \in T$. By (a) there is a $t \in T$ such that $t_{1} t_{2}=e t$. So $t_{1} t_{2}=e t=e^{2} t=e(e t)=e\left(t_{1} t_{2}\right)=\left(e t_{1}\right) t_{2}=\left(e t_{1} e\right) t_{2}=\left(e t_{1}\right)\left(e t_{2}\right)=p\left(t_{1}\right) p\left(t_{2}\right)$.

The surjective part of an action

Definition 1.5. If $T$ is a semigroup acting on $X$ we say that an element $t \in T$ is surjective provided

$$
(\forall a \in X)(\exists x \in X) \quad a=t \cdot x
$$

The set of all surjective elements will be denoted by $\Sigma(T, X)$.

We recall that in a semigroup $T$, the $R$-class $R(t)$ of an element $t$ consists of all $t^{\prime} \in T$ for which there are elements $u, v \in T$ such that $t=t^{\prime} u$ and $t^{\prime}=t v$. For an idempotent $e$, the largest subgroup containing $e$ is denoted $H(e)$. Notice $H(e) \subseteq R(e)$.

If $T$ is a semigroup we denote its set of idempotents by $E(T)$.

Lemma 1.6. Suppose that $(T, e)$ acts e-effectively on $X$. Then

(i) $\Sigma(T, X)$ is a subsemigroup of $T$ containing $R(e)$.

(ii) $\Sigma(S, X)=\Sigma(T, X) \cap S$ is a subsemigroup of the structure monoid $S$ containing $R(e) \supseteq H(e)$.

(iii) $E(\Sigma(S, X))=\{e\}$.

(iv) If $h \in H(e)$ then the function $\sigma: X \rightarrow X, \sigma(x)=h \cdot x$ is bijective with inverse given by $\sigma^{-1}(x)=h^{-1} \cdot x$.

Proof. (i) Take $s_{1}, s_{2} \in \Sigma(T, X)$ and pick $a \in X$. First find an $x_{1}$ such that $a=s_{1} \cdot x_{1}$. Next find $x \in X$ such that $x_{1}=s_{2} \cdot x$. Then $a=s_{1} \cdot\left(s_{2} \cdot x\right)=\left(s_{1} s_{2}\right) \cdot x$. This proves that $\Sigma(T, X)$ is a subsemigroup of $T$. Now suppose that $r \in R(e)$. Then there is a $t \in T$ such that $e=r t$. If $a \in X$ is given, set $x=t \cdot a$. Then $r \cdot x=r \cdot(t \cdot a)=r t \cdot a=e \cdot a=a$. Thus $r$ is surjective.

(ii) is a consequence of (i).

(iii) By (i) and (ii) we know $e \in E(\Sigma(S, X))$. Conversely, suppose now that $e^{\prime} \in E(\Sigma(S, X))$. Define $\tau: X \rightarrow X$ by $\tau(x)=e^{\prime} \cdot x$. Since $e^{\prime}$ is idempotent, $\tau$ is a retraction. Since $e^{\prime} \in \Sigma(T, X)$, then $\tau$ is surjective. A surjective retraction is the identity map. Thus $e^{\prime} \cdot x=x$ for all $x \in X$. But also $x=e \cdot x$. Hence $e$-effectivity (3) implies $e e^{\prime}=e e=e$. But also $e^{\prime} \in S=e T$. Hence there is a $t \in T$ such that $e^{\prime}=e t$. Thus $e e^{\prime}=e e t=e t=e^{\prime}$. Therefore $e=e^{\prime}$ and this proves the claim.

(iv) $h^{-1} \cdot \sigma(x)=h^{-1} \cdot(h \cdot x)=h^{-1} h \cdot x=e \cdot x=x$. Similarly, $\sigma\left(h^{-1} \cdot x\right)=x$.

If $T$ is an inflation of $S=e T$, then $E(\Sigma(T, X))=\{e\}$. Otherwise this need not be the case.

The remark on the idempotents has a noteworthy consequence for compact actions which we shall see presently. 
Lemma 1.7. If $X$ is a compact Hausdorff space and $T$ a topological semigroup with an idempotent $e$ acting e-effectively on $X$, and if $S=\epsilon T$, then $S$ is closed in $T$ and $\Sigma(S, X)$ is closed in $S$.

Proof. Retracts of Hausdorff spaces are closed, and $S=e T$ is a retract. Hence $S$ is closed in $T$.

Now let $s \in \overline{\Sigma(S, X)}$. Then there is a net $s_{j} \in \Sigma(S, X)$ converging to $s$. We claim that $s$ is surjective. Let $a \in X$. Since $s_{j}$ is surjective for each $j$, there is an $x_{j} \in X$ such that $a=s_{j} \cdot x_{j}$. As $X$ is compact we find a subnet $x_{j(k)}$ converging to some $x \in X$. Since the action $(t, x) \mapsto t \cdot x: T \times X \rightarrow X$ is continuous, $s \cdot x=\lim _{k} s_{j(k)} \cdot x_{j(k)}=a$. Thus $s$ is surjective as asserted.

Remark 1.8. Under the circumstances of Lemma 1.7, if $T$ is a compact Hausdorff space, then so is $S=e T$.

Proof. By 1.7 the space $S$ is closed in a compact Hausdorff space. p. 15):

The following is a basic and classical fact on compact monoids (cf. [3], 1.19,

Proposition 1.9. Any compact monoid with but one idempotent is a group.

This allows us to conclude the following

Proposition 1.10. Suppose that a compact semigroup $(T, e)$ with an idempotent $e$ acts e-effectively on a compact Hausdorff space $X$. Then

$$
\Sigma(S, X)=H(e)
$$

Proof. By Lemma 1.8 and Lemma 1.6(iii), $\Sigma(S, X)$ is a compact monoid with one idempotent. Hence it is a group by Proposition 1.9. Consequently, $\Sigma(S, X) \subseteq H(e)$. The reverse inclusion holds generally by Lemma 1.6(ii).

Actions with constants

For any $X$ we introduce a notation for the constant self-maps of $X$ as follows: $\kappa_{x}: X \rightarrow X, \kappa_{x}(y)=x$. Trivially, in a topological space the constant self-maps are continuous.

Definition 1.11. We say that a topological semigroup with idempotent $(T, e)$ acts on a topological space $X$ with constants if there is a continuous function $\kappa: X \rightarrow T$ such that

$$
\left(\forall x, x^{\prime} \in X\right) \quad \kappa(x) \cdot x=x
$$

Clearly, the maps $x^{\prime} \mapsto \kappa(x) \cdot x^{\prime}$ are exactly the constant self-maps $\kappa_{x}$.

Examples are readily at hand: let $X$ be a compact Hausdorff space and $T$ the topological Hausdorff monoid $\mathcal{C}(X, X)$ of all continuous self-maps with the compact-open topology. Its identity is $e=\mathrm{id}_{X}$. The monoid $T=e T=S$ acts via $(\phi, x) \mapsto \phi(x): T \times X \rightarrow X$ with constants via $\kappa: X \rightarrow S$ given by $\kappa(x)=\kappa_{x}$. 
Remark 1.12. Suppose that the conditions of 1.11 are satisfied. Then the function $\kappa^{\prime}: X \rightarrow S$ defined by $\kappa^{\prime}(x)=e \kappa(x)$ satisfies

$$
\left(\forall x, x^{\prime} \in X\right) \kappa^{\prime}(x) \cdot x^{\prime}=x .
$$

Proof. We compute $\kappa^{\prime}(x) \cdot x^{\prime}=e \kappa(x) \cdot x^{\prime}=e \cdot\left(\kappa(x) \cdot x^{\prime}\right)=e \cdot x=x$.

of $\kappa$ is $S$.

It therefore would be no loss of generality to assume outrightly that the range

Lemma 1.13. Suppose that $(T, e)$ acts effectively on $X$ with constants. Then we have the following conclusions:

(i) $\kappa$ is injective and is a morphism of $S$-actions, i.e.,

$$
(\forall s \in S, x \in X) \kappa(s \cdot x)=s \kappa(x) \text {. }
$$

(ii) $\kappa(X)=M(S)$ is a minimal ideal of the structure monoid $S=e T$ consisting of left zeroes. It is the minimal ideal of $T$ if $e$ is central.

(iii) Let $\kappa_{0}: X \rightarrow M(S)$ be the corestriction of $\kappa$ to its image. Then $\kappa_{0}$ is an isomorphism of $S$-actions.

Proof. (i) $\kappa(x)=\kappa(y)$ implies $x=\kappa(x) \cdot x=\kappa(y) \cdot x=y$ which proves that $\kappa$ is injective. For all $x, y \in X$ and $s \in S$ we have $(s \kappa(x)) \cdot y=s \cdot(\kappa(x) \cdot y)=s \cdot x=$ $\kappa(s \cdot x) \cdot y$. So $s \kappa(x)=\kappa(s \cdot x)$. This proves $(8)$.

(ii) We take $x, y \in X, s \in S$. Then $(\kappa(x) s) \cdot y=\kappa(x) \cdot(s \cdot y)=x=\kappa(x) \cdot y$. Since $y$ is arbitrary and $\kappa(x)$ and $\kappa(x) s$ are in $S$, the effectivity of the action yields $\kappa(x) s=\kappa(x)$. Thus $\kappa(X)$ consists of left zeroes and therefore $\kappa(X) \subseteq M(S)$. By (i) $S \kappa(X) \subseteq \kappa(X) \subseteq S$. Thus $\kappa(X)$ is a left ideal of $S$, and hence an ideal since it consists of left zeroes. This implies $\kappa(X)=M(S)$ as asserted in (ii). If $e$ is central, then $S$ is an ideal of $T$ and the computations above are valid for $s \in T$ since then $\kappa(x) s \in S$ even in that case.

(iii) is a trivial consequence of (i).

Corollary 1.14. If $(T, e)$ acts e-effectively on $X$, then

$$
\operatorname{card} X=\operatorname{card} M(S) \leq \operatorname{card} S .
$$

\section{Functional Algebras}

Definition 2.1. A functional algebra is a pair of topological spaces $(T, X)$ together with the following structural data:

(i) $T \subseteq X$ is a subspace endowed with a topological semigroup multiplication $(x, y) \mapsto x \circ y: T \times T \rightarrow T$ and with an idempotent $e$. The monoid $S \stackrel{\text { def }}{=}$ $(e \circ T, \circ)$ with identity $e$ will be called the structure monoid of the functional algebra $X$.

(ii) There is an e-effective continuous action $(x, y) \mapsto x \cdot y: T \times X \rightarrow X$.

(iii) There is a distinguished element $y \in T$ such that

$$
(\forall x \in X) x \cdot(y \cdot x)=y \cdot x .
$$

(iv) There is a continuous function $\kappa: X \rightarrow X, \kappa(X) \subseteq T$ satisfying

$$
\left(\forall x, x^{\prime} \in X\right) \kappa(x) \cdot x^{\prime}=x .
$$


Remark 2.2. Suppose that the conditions of 2.1 are satisfied. Then the element $y^{\prime}=e \circ y \in S$ satisfies

$$
(\forall x \in X) x \cdot\left(y^{\prime} \cdot x\right)=y^{\prime} \cdot x .
$$

Proof. $\quad x \cdot\left(y^{\prime} \cdot x\right)=x \cdot([e \circ y] \cdot x)=x \cdot(e \cdot(y \cdot x))=x \cdot(y \cdot x)=y \cdot x=e \cdot(y \cdot x)=[e \circ y] \cdot x=$ $y^{\prime} \cdot x$.

So it would be no loss of generality to postulate that $y$ is in $S$ outrightly. By 1.12 it also would be no loss of generality to assume in 2.1 (iv) that $\kappa(X) \subseteq S$.

Remark 2.3. In a functional algebra,

$$
\operatorname{card} X=\operatorname{card} T=\operatorname{card} S .
$$

Proof. By Corollary 1.14, card $X=\operatorname{card} M(S) \leq \operatorname{card} S$. Since $S \subseteq T \subseteq X$ we have $\operatorname{card} S \leq \operatorname{card} T \leq \operatorname{card} X$. The assertion follows.

Lemma 2.4. Let $(T, X)$ be a functional algebra. Then

$$
(\forall x \in X) x=y \cdot \kappa(x) \text {. }
$$

As a consequence, $y \in \Sigma(T, X)$ and $e$ o $y \in \Sigma(S, X)$.

Proof. In view of the definition of $\kappa$ in $(7)$ we compute $x=\kappa(x) \cdot(y \cdot \kappa(x))=y \cdot \kappa(x)$ by (9). This proves (11). The definition of surjectivity together with (11) directly shows $y \in \Sigma(T, X)$. By 2.2, the element $e \circ y$ has the same properties as $y$ and is contained in $e \circ T=S$, and thus $e \circ y \in \Sigma(T, X) \cap S=\Sigma(S, X)$.

We say that a functional algebra $(T, X)$ is degenerate if $X$ is singleton.

Lemma 2.5. Any functional algebra $X$ with $y \in H(e)$ is degenerate.

Proof. If $y \in H(e)$ then $x \mapsto y^{-1} \cdot x$, where $y^{-1}$ is the inverse of $y$ in $H(e)$ is bijective by Lemma $1.6\left(\right.$ iv). Thus $y^{-1} \cdot X=X$. Now Lemma 2.4 implies $y^{-1} \cdot X=$ $y^{-1} \cdot(y \cdot \kappa(X))=\left(y^{-1} \circ y\right) \cdot \kappa(X)=e \cdot \kappa(X)=\kappa(X)$. By 1.13(ii) we have $\kappa(X)=M(S)$. Hence $X=M(S)$. In particular $e \in M(S)$, and $M(S)$ consists of left zeroes. Thus the identity $e$ of $S$ is a left zero. This implies $S=\{e\}$. On the other hand, $\kappa$ maps $X$ injectively onto $M(S)$ by $1.13(\mathrm{i})$. Thus $X=\{e\}$.

Definition 2.6. By a compact functional algebra we mean a functional algebra $(T, X)$ such that $S=e \circ T$ and $X$ are compact Hausdorff spaces.

Theorem 2.7. Any compact functional algebra is degenerate.

Proof. By Proposition 1.10 we have $\Sigma(S, e \cdot X)=H(e)$. By 2.4, e o $y \in \Sigma(S, X)$. Hence $e \circ y \in H(e)$. Then Remark 2.2 and Lemma 2.5 finish the proof.

Frequently the axiom (iv) of a functional algebra securing constant self-maps of $X$ in $T$ in a continuous fashion is realized by a stronger axiom.

Definition 2.8. A functional algebra $(T, X)$ is a $k$-functional algebra iff the following condition is satisfied:

(iv') There is an element $k \in T$ such that

$$
\left(\forall x, x^{\prime} \in X\right) \quad(k \cdot x) \cdot x^{\prime}=x .
$$

We note by setting $\kappa(x)=k \cdot x$ that condition (iv') implies (iv). 
Remark 2.9. Suppose that $(T, X)$ is a $k$-functional algebra. Then the element $k^{\prime}=\epsilon \circ k \in S=\epsilon \circ T$ satisfies

$$
\left(\forall x, x^{\prime} \in X\right) \quad\left(k^{\prime} \cdot x\right) \cdot x^{\prime}=x .
$$

Proof. $\quad\left(k^{\prime} \cdot x\right) \cdot x^{\prime}=((e \circ k) \cdot x) \cdot x^{\prime}=\left(e \cdot(k \cdot x) \cdot x^{\prime}=(k \cdot x) \cdot x^{\prime}=x\right.$ by $1.1(1)$ and $2.8\left(7^{\prime}\right)$.

It is therefore no loss of generality to assume right away that $k \in S$. So, in view of Remark 2.2 above we make the following definition:

Definition 2.10. A strict functional algebra is a functional $k$-algebra with $k, y \in$ $S=e \circ T$.

Lemma 2.11. The elements $y$ and $k$ of the structure monoid $S$ of a strict functional algebra satisfy the relation $y \circ k=e$.

Proof. We set $\kappa(x)=k \cdot x$; then $\left(7^{\prime}\right)$ implies (7). From Lemma 2.4 we then deduce

$$
(\forall x \in X) \quad x=y \cdot(k \cdot x) \text {. }
$$

But $y \cdot(k \cdot x)=(y \circ k) \cdot x$ by $1.1(1)$. Thus $(y \circ k) \cdot x=x=e \cdot x$ for all $x$. Since the action is $e$-effective and since $y \circ k \in S=e \circ T$, we conclude $y \circ k=e \circ(y \circ k)=\epsilon \circ \epsilon=\epsilon$

In view of Lemma 2.11 we recall that the free monoid generated by two elements $p$ and $q$ subject to the relation $p q=e$ is called the bicyclic semigroup $B(p, q)$ (cf. [1], pp. $43-46$ ). The homomorphic images of $B(p, q)$ are either isomorphic to $B(p, q)$, or else they are cyclic groups.

Proposition 2.12. If $(X, T)$ is a nondegenerate strict functional algebra then the structure monoid $S=e \circ T$ contains a bicyclic semigroup $B$ as submonoid.

Proof. We let $B$ be the submonoid generated by $k$ and $y$. By way of contradiction we suppose that $B$ is not a bicyclic semigroup. Then $B$ is a proper homomorphic image of a bicyclic semigroup and is therefore a group. Since $e=y \circ k \in B$ by Lemma 2.11, this implies $y \in B \subseteq H(e)$. Then Lemma 2.5 shows that $X$ is degenerate in contradiction with our hypothesis.

In the theory of compact topological semigroups it is well-known that no compact topological semigroup can contain a bicyclic semigroup (see e.g. [5]). This yields a proof of the fact that every compact strict functional algebra is degenerate which also follows immediately from Theorem 2.7.

It would be reasonable to speculate that degeneracy theorems for strict functional algebras will have to rely on some proof that bicyclic semigroups cannot exist as submonoids in $S$.

A simple example of $B(p, q)$ acting on a set is given by the operators $P: \mathbb{N} \rightarrow$ $\mathbb{N}$ and $Q: \mathbb{N} \rightarrow \mathbb{N}$ by $P(n)=\max \{1, n-1\}$ and $Q(n)=n+1$. A similar example can be constructed on the space of nonnegative real numbers.

Exercise 2.13. (i) For each $r \in \mathbb{R}$ the function $x \mapsto x-r$ is an automorphism of the sup-semilattice $(\mathbb{R}, \vee)$. The map $r \mapsto(x \mapsto x-r):(\mathbb{R},+) \rightarrow \operatorname{Aut}(\mathbb{R}, \mathcal{V})$ is a homomorphism $\alpha$.

(ii) The semidirect product $S=(\mathbb{R}, \vee) \rtimes_{\alpha}(\mathbb{R},+)$ is a topological semigroup with respect to the multiplication $(x, r)\left(x^{\prime}, r^{\prime}\right)=\left(x \vee\left(x^{\prime}-r\right), r+r^{\prime}\right)$. The projection $(x, r) \mapsto r: S \rightarrow(\mathbb{R},+)$ is a homomorphism,

(iii) We set $\mathbb{Z}^{+}=\{0,1,2, \ldots\}$ and $\mathbb{R}^{+}=\{r \in \mathbb{R} \mid r \geq 0\}$. The subset $B(p, q)=\mathbb{Z}^{+}(1,-1)+\mathbb{Z}^{+}(0,1)$ is a subsemigroup of $S$ generated by $p=(0,1)$ and $q=(1,-1)$ and isomorphic to the bicyclic semigroup.

The subset $C(p, q)=\mathbb{R}^{+}(1,-1)+\mathbb{R}^{+}(1,-1)$ is a monoid, the continuous bicyclic semigroup. 


\section{Functional Domains, Functional Monoids, and Environment Models}

In this section, we review of the concepts of functional domains, functional monoids and environment models which were discussed in [6], and interpret our degeneracy results in these settings. However, we base our considerations on an arbitrary cartesian closed category with function spaces. We assume some knowledge of the basic aspects of cartesian closed categories presented in the Appendix. In particular, we utilize the notation used there.

In [4], MEYER defines a functional domain to be a set $X$ together with a family of self-maps $[X \rightarrow X]$ which is a retract of $X$ (in $\mathcal{S E \mathcal { T }}$ ). Since we are concerned with such objects in various categories, we have rephrased his definition to include the possibility of objects in arbitrary cartesian closed categories.

Throughout this section we shall fix a concrete cartesian closed category $\mathcal{C}$ with function spaces. (See. A.11 and A.13.)

Definition 3.1. (i) If $X$ is an object in a cartesian closed category $\mathcal{C}$, then a functional domain on $X$ is formally a triple $([X \rightarrow X], j, p)$ consisting of a $\mathcal{C}$ subobject of $X^{X}$, together with an embedding-projection pair $(j, p)$ of $\mathcal{C}$-morphisms

$$
\begin{aligned}
& j:[X \rightarrow X] \rightarrow X \\
& p: X \rightarrow[X \rightarrow X] .
\end{aligned}
$$

I.e., $p j=\mathrm{id}_{X}$.

(ii) A functional monoid on the object $X$ of $\mathcal{C}$ is a quadruple $(M,[X \rightarrow$ $X], j, p)$ such that $([X \rightarrow X], j, p)$ is a functional domain with a submonoid $M$ of $X^{X}$ such that the inclusion morphism $M \rightarrow X^{X}$ factors through the inclusion $[X \rightarrow X] \rightarrow X^{X}$. If the constant-picker $\kappa_{X}^{X}: X \rightarrow X^{X}$ (see A.4) factors through the inclusion $M \rightarrow X^{X}$, then we say the monoid $M$ has constants.

(iii) We say that a functional domain $([X \rightarrow X], j, p)$ is standard if $[X \rightarrow X]$ is a submonoid of $X^{X}$. Then $([X \rightarrow X],[X \rightarrow X], j, p)$ is called the associated standard functional monoid. If a functional domain $([X \rightarrow X], j, p)$ is such that the inclusion $[X \rightarrow X] \rightarrow X^{X}$ is an isomorphism, then it is called special. Special functional domains always have an associated standard functional monoid.

Functional domains in standard topological categories

Our main application will deal with a concrete cartesian closed category $\mathcal{C}$ based in the category of topological spaces.

Definition 3.2. We say that $\mathcal{C}$ is a standard topological category if the following hypotheses are satisfied:

(i) All objects of $\mathcal{C}$ are (among other things) topological spaces and morphisms are continuous maps. (That is, there is a faithful functor into the category $\mathcal{T O P}$ of topological spaces and continuous maps through which the grounding functor $|\cdot|: \mathcal{C} \rightarrow \mathcal{S E \mathcal { T }}$ factors.)

(ii) $\mathcal{C}$ is a concrete cartesian closed category with function spaces (see A.1, A.11, A.13).

Thus in a standard topological category, every object $X$ has an underlying set $|X|$, the terminal objects are the singletons, there is a natural bijection $j_{A}: \mathcal{C}(\{*\}, X) \mapsto|X|$ given by $j_{A}(f)=|f|(*)$. If no confusion is possible we shall omit the vertical bars. However, there are instances where it is indispensable to use 
them. If $X$ and $Y$ are spaces in a standard topological category, then $Y^{X}$ is an object in the category whose underlying set $\left|X^{Y}\right|$ may be viewed as $\mathcal{C}(X, Y)$. If $\mathcal{C}$ has arbitrary products, which is the case in most of the categories we consider, then $Y^{|X|}$ is a product in $\mathcal{C}$. If the grounding functor preserves products (normally the case!) then $\mid Y^{|X|}$ may be identified with $|Y|^{|X|}$ the set of all functions from $|X|$ to $|Y|$.

By definition, in view of A.13, the constant self-maps $\kappa_{x}: X \rightarrow X, \kappa_{x}\left(x^{\prime}\right)=x$ are all members of $\left|X^{X}\right|$.

The most familiar example of a standard topological category is the category of Hausdorff $k$-spaces with the $k$-product and $X^{Y}$ the space of all continuous functions $X \rightarrow Y$ endowed with the compact-open topology and given the $k$-refinement.

The lambda calculus.

The whole point of studying functional domains and functional monoids is to understand what categories give rise to models of the untyped lambda calculus. We now formally introduce the calculus and then relate it to the notion of a functional monoid.

Suppose that $V$ is a set of elements called variables, and that $C$ is a set of elements called constants. Then the untyped lambda calculus has syntax given by the following $\mathrm{BNF}$ :

$$
p::=c|v| p p \mid \lambda v \cdot p
$$

where $c \in C$ and $v \in V$.

We shall denote the set of all lambda terms (including the elements of $V$ and $C)$ by $\Lambda$. Instead of $\lambda v_{1} \cdot\left(\ldots\left(\lambda v_{n} . m\right) \ldots\right)$ we shall write $\lambda v_{1} \ldots v_{n} . m$, as is usual in this context.

For the sake of the record we recall that usually one denotes the substitution of the term $q$ for the variable $v$ occurring freely in the term $m$ by $m[q / v]$ and imposes the following reduction rules:

( $\alpha) \lambda v . m=\lambda u . m[u / v]$ for $u$ not free in $m$,

( $\beta)(\lambda v . m) q=m[q / v]$, and

( $\eta) \lambda v \cdot(m v)=m$ for $v$ not free in $m$.

We then say that a term $p$ converts to a term $q$ if the application of a (sequence of the) rule(s) can be applied to $p$ to obtain $q$. Note that conversion is a symmetric relation, and we assume that it also is reflexive.

However, we emphasize that here $\Lambda$ is the free algebra generated by $C \cup V$ via the binary operation $(m, n) \mapsto m n$ and the family of unary operations $m \mapsto \lambda v \cdot m$, $v \in V$. This will be important in our recursive definition of functions on $\Lambda$.

The lambda calculus supports composition as a "derived" operator, as follows:

Definition 3.3. Define the operator

$$
\text { compose: } \Lambda \times \Lambda \rightarrow \Lambda \text { by } \operatorname{compose}(m, n) \stackrel{\text { def }}{=} \lambda x \cdot m(n x),
$$

where $x$ is not free in either $m$ or $n$.

Lemma 3.4. For $m_{1}, m_{2}, m_{3} \in \Lambda$, if $\beta$ reduction is imposed,

$$
m_{1}\left(m_{2} m_{3}\right) \stackrel{\beta}{=} \operatorname{compose}\left(m_{1}, m_{2}\right) m_{3} \text {. }
$$

Proof. We compute compose $\left(m_{1}, m_{2}\right)\left(m_{3}\right)=\left(\lambda v \cdot m_{1}\left(m_{2} v\right)\right) m_{3}=m_{1}\left(m_{2} m_{3}\right)$ by $\beta$-reduction. 
As a last comment, we define the following special elements of the algebra $\Lambda \Lambda:$

$$
\begin{aligned}
\mathbf{K} & =\lambda x y \cdot x, \\
\mathbf{S} & =\lambda x y z \cdot x z(y z), \\
\mathbf{Y} & =\lambda f \cdot(\lambda v \cdot f(v v))(\lambda v \cdot f(v v)), \\
\mathbf{I} & =\lambda x \cdot x .
\end{aligned}
$$

The element $\mathbf{Y} \in \Lambda$ is called the paradoxical combinator. With $\beta$-conversion we have $\mathbf{I} \cong(\mathrm{SK}) \mathbf{K}$.

\section{Construction of an environment model}

Since $\Lambda$ is a free algebra we can define the length of a $\lambda$ term. The length function $\ell: \Lambda \rightarrow \mathbb{N}$ is defined inductively as follows:

(i) $\ell(m)=1$ if $m \in C \cup V$.

(ii) $\ell(m n)=\ell(m)+\ell(n)$.

(iii) $\ell(\lambda v \cdot m)=\ell(m)+1$

We shall write

$$
\Lambda_{i}=\{m \in \Lambda: \ell(m) \leq i\} .
$$

This defines an ascending filtration $\Lambda_{1} \subseteq \Lambda_{2} \subseteq \cdots$ with $\Lambda=\bigcup_{i=1}^{\infty} \Lambda_{i}$ which we shall use for recursive definitions of functions with $\Lambda$ as domain and a suitable codomain.

Suppose now that we are given a functional domain $([X \rightarrow X], j, p)$ over the object $X$ in $\mathcal{C}$ (cf. Definition 3.1). We consider the underlying set $|X|$ of $X$ as the set of constants in $\Lambda$ so that in fact we may write $|X| \subseteq \Lambda$. The functions $\rho: V \rightarrow|X|$ are called environments; therefore, we introduce a set Env $=|X|^{V}$. Recall that by Definition A.11 the category $\mathcal{C}$ has products and that the grounding functor preserves products. Accordingly, suppressing a natural isomorphism, we have

$$
\text { Env }=|X|^{V}=\left|X^{V}\right| \text { for the } \mathcal{C} \text {-object } X^{V} .
$$

Each environment $\rho: V \rightarrow|X|$ assigns to each variable $v$ a constant $\rho(v) \in|X|$ as value. Also, the power $X^{\text {Env }}$ is a well-defined $\mathcal{C}$-object. We recall (from A.14 and a subsequent remark (\#)) that there is a natural injective morphism

$$
\gamma_{X}^{\left(X^{V}\right)}: X^{\left(X^{v}\right)} \rightarrow X^{\left|X^{v}\right|}=X^{\mathrm{Env}} .
$$

Thus, in view of $\left|X^{\left(X^{V}\right)}\right|=\mathcal{C}\left(X^{V}, X\right)$ we obtain an inclusion map

$$
\left|\gamma_{X}^{\left(X^{v}\right)}\right|: \mathcal{C}\left(X^{V}, X\right) \rightarrow\left|X^{\text {Env }}\right|=|X|^{\text {Env }} .
$$

In this sense we write

$$
\mathcal{C}\left(X^{V}, X\right) \subseteq|X|^{\text {Env }} .
$$

From these data we construct a function $\mathcal{V}: \Lambda \rightarrow \mathcal{C}\left(X^{V}, X\right) \subseteq|X|^{\text {Env }}$ recursively w.r.t the length $\ell$ in four steps (a), (b), (c), and (d). More specifically, we shall define functions

$$
\mathcal{V}_{i}: \Lambda_{i} \rightarrow \mathcal{C}\left(X^{V}, X\right), \quad i=1,2, \ldots
$$

such that $\mathcal{V}_{i+1} \mid \Lambda_{i}=\mathcal{V}_{i}$, and then we define $\mathcal{V}: \Lambda \rightarrow \mathcal{C}\left(X^{V}, X\right)$ by $\mathcal{V}[m]=\mathcal{V}_{i}[m]$ unambiguously with $i \geq \ell(m)$. 
(a) $(\forall x \in|X| \subseteq \Lambda, \rho \in \operatorname{Env}) \quad \mathcal{V}_{1}[x] \rho=x$,

i.e., $\mathcal{V}_{1}[x] \in \mathcal{C}\left(X^{V}, X\right)$ is the constant function with value $x$.

(b) $(\forall v \in V, \rho \in \operatorname{Env}) \quad \mathcal{V}_{1}[v] \rho=\rho(v)$, i.e., on $V$ the function $\mathcal{V}_{1}$ agrees with the evaluation function, or projection, $V \rightarrow \mathcal{C}\left(X^{V}, X\right)$.

Thus $\mathcal{V}_{1}: \Lambda_{1} \rightarrow \mathcal{C}\left(X^{V}, X\right)$ is defined. Now suppose that the function $\mathcal{V}_{i}: \Lambda_{i} \rightarrow X^{\text {Env }}$ has been defined on the set $\Lambda_{i}$ of lambda terms of length $\leq i$ such that $\mathcal{V}_{j+1} \mid \Lambda_{j}=\mathcal{V}_{j}$ for $j=1, \ldots, i-1$. We recall that we have a binary operation $(x, y) \mapsto x \cdot y: X \rightarrow X$ given by $x \cdot y=p(x)(y)$ (cf. 3.2(ii)). If $\mu \in \Lambda$ with $\ell(\mu)=i+1$, then, since $\Lambda$ is a free algebra, either $\mu=m n$ with uniquely defined $\lambda$-terms $m$ and $n$ such that $\max \{\ell(m), \ell(n)\} \leq i$, or $\mu=\lambda v \cdot m$ with a uniquely defined $\lambda$-term $m$ such that $\ell(m)=i$. The first case is handled rather simply, the second will ask for greater circumspection. In the first case we define

$$
\text { (c) }\left(\forall m, n \in \Lambda_{i}, \rho \in \mathrm{Env}\right) \quad \mathcal{V}_{i+1}[m n] \rho=\left(\mathcal{V}_{i}[m] \rho\right) \cdot\left(\mathcal{V}_{i}[n] \rho\right) \text {. }
$$

Since the map $(x, y) \mapsto x \cdot y=p(x)(y): X \times X \rightarrow X$ is a $\mathcal{C}$-morphism, then $\mathcal{V}_{i+1}[m n] \in$ $\mathcal{C}\left(X^{V}, X\right)$ by $(\mathrm{c})$, since $\mathcal{V}_{i}[m], \mathcal{V}_{i}[n] \in \mathcal{C}\left(X^{V}, X\right)$.

In order to deal with the harder second case, we note that for each environment $\rho: V \rightarrow X$, each constant $x \in X$, and each variable $v$ we can define a new environment $\rho\{x / v\}: V \rightarrow X$ by setting

$$
\rho\{x / v\}(w)= \begin{cases}\rho(w), & \text { if } w \neq v, \\ x, & \text { if } w=v .\end{cases}
$$

Note that $\rho\{x / u\}\{y / v\}(w)=y$ if $w=v$, while

$$
\rho\{x / u\}(w)= \begin{cases}\rho(w), & \text { if } w \neq u, \\ x, & \text { if } w=u .\end{cases}
$$

Thus

$$
\rho\{x / u\}\{y / v\}(w)= \begin{cases}\rho(w), & \text { if } v \neq w \neq u \\ x, & \text { if } w=u \text { and } w \neq v \\ y, & \text { if } w=v\end{cases}
$$

It follows that

$$
\rho\{x / u\}\{y / v\}= \begin{cases}\rho\{y / v\}\{x / u\} & \text { if } u \neq v \\ \rho\{y / v\} & \text { if } u=v\end{cases}
$$

Let us fix $v \in V$. The equality $V=(V \backslash\{v\}) \cup\{v\}$ allows us to write $X^{V}=X^{V \backslash\{v\}} \times X$. Setting $\rho=\left(\xi_{w}\right)_{w \in V}$ we define $\Omega_{v}(x): X^{V} \rightarrow X^{V}$ by

$$
\Omega_{v}(x)(\rho)=\Omega_{v}(x)\left(\left(\xi_{w}\right)_{w \in w \in V \backslash\{v\}}, x^{\prime}\right)=\left(\left(\xi_{w}\right)_{w \in w \in V \backslash\{v\}}, x\right)=\rho\{x / v\} .
$$

Using 3.3(iii) (see also A.4(i)) and $K(x)=p j\left(\kappa_{x}\right)$, it follows that $K: X \rightarrow X^{X}$ is a morphism and we have

$$
\Omega_{v}(x)\left(\left(\xi_{w}\right)_{w \in w \in V \backslash\{v\}}, x^{\prime}\right)=\left(\left(\xi_{w}\right)_{w \in w \in V \backslash\{v\}}, K(x)\left(x^{\prime}\right)\right) .
$$

Thus, in this notation,

$$
\left(x, \Omega_{v}\right) \mapsto \Omega_{v}(x): X \times X^{V} \rightarrow X^{V}
$$


is a $\mathcal{C}$-morphism. The universal property of cartesian closed categories (see A.2) then shows that $\Omega_{v}: X \rightarrow\left(X^{V}\right)^{\left(X^{V}\right)}$ is a morphism, i.e.,

$$
\Omega_{v} \in \mathcal{C}\left(X,\left(X^{V}\right)^{\left(X^{V}\right)}\right)
$$

Note that the composition

$$
\circ: X^{\left(X^{V}\right)} \times\left(X^{V}\right)^{\left(X^{V}\right)} \rightarrow X^{\left(X^{V}\right)}
$$

is a morphism (see A.4(ii)). Hence, if $F \in \mathcal{C}\left(X^{V}, X\right)$, then for each $v \in V$ the function $x \mapsto F \circ \Omega_{v}(x): X \rightarrow X^{\left(X^{V}\right)}$ is a $\mathcal{C}$-morphism. We note $\left(F \circ \Omega_{v}(x)\right)(\rho)=$ $F(\rho\{x / v\})$ and that $x \mapsto F(\rho(x / v)): X \rightarrow X$ is a $\mathcal{C}$-morphism for every $\rho \in|X|^{V}=$ Env. Also, setting

$$
\Phi_{\rho v} \in\left|X^{X}\right|=\mathcal{C}(X, X) \quad \text { by } \quad \Phi_{\rho v}(x)=\Omega_{v}(x)(\rho),
$$

we note that

$$
\rho \mapsto F \circ \Phi_{\rho v}: X^{V} \rightarrow X^{X}
$$

is a $\mathcal{C}$-morphism.

In order to complete our definition of $\mathcal{V}_{i+1}$ we must take recourse to a definition as follows:

Definition 3.5. The functional domain $([X \rightarrow X], j, p)$ in $\mathcal{C}$ is called an environment model in $\mathcal{C}$ provided the following recursive condition is satisfied for $i=1,2,3, \ldots$ :

Suppose that $\mathcal{V}_{i}: \Lambda_{i} \rightarrow \mathcal{C}\left(X^{V}, X\right)$ is defined in such a fashion that (a) and (b) are satisfied as well as (c) for $\ell(m)+\ell(n) \leq i$. Suppose further that in the case $i \geq 2$, for all $m \in \Lambda_{i-1}$ and all $(v, \rho) \in V \times \operatorname{Env}$ we have

$$
\mathcal{V}_{i}[m] \circ \Phi_{\rho v} \in[X \rightarrow X]
$$

Then for all $m \in \Lambda_{i}$ and all $(v, \rho) \in V \times$ Env we have

$$
\mathcal{V}_{i}[m] \circ \Phi_{\rho v} \in[X \rightarrow X]
$$

Note that $\left[\mathcal{V}_{i}[m] \circ \Omega_{v}(x)\right] \rho=\mathcal{V}_{i}[m] \rho\{x / v\}$ for all $(v, \rho) \in V \times$ Env, and that $p(X)=[X \rightarrow X]$. Thus, saying that $([X \rightarrow X], j, p)$ is an environment model means this: whenever $m \in \Lambda_{i}$ and $\mathcal{V}_{i}[m] \in \mathcal{C}\left(X^{V}, X\right)$ has been defined as specified, then $\left(x \mapsto \mathcal{V}_{i}[m] \rho\{x / v\}\right) \in p(X)$ for all $(v, \rho) \in V \times$ Env . can define

Now suppose that $([X \rightarrow X], j, p)$ is an environment model in $\mathcal{C}$. Then we

(d) $\mathcal{V}_{i+1}[\lambda v . m](\rho)=j\left(\mathcal{V}_{i}[m] \circ \Phi_{\rho v}\right)$ for all $m \in \Lambda_{i}$ and all $(\rho, v) \in$ Env $\times V$.

Since $\left.\rho \mapsto\left\{x \mapsto F \circ \Omega_{v}(x)\right)(\rho)=F(\rho\{x / v\})\right\}$ is in $\mathcal{C}\left(X^{V}, X\right)$ for each $F \in \mathcal{C}\left(X^{V}, X\right)$ we know that $\mathcal{V}_{i+1}[\lambda v . m] \in \mathcal{C}\left(X^{V}, X\right)$.

In any environment model, we have now recursively defined functions $\mathcal{V}_{i}: \Lambda_{i} \rightarrow \mathcal{C}\left(X^{V}, X\right) \subseteq|X|^{\text {Env }}$ such that $V_{i+1} \mid \Lambda_{i}=\mathcal{V}_{i}$ for $i=1,2, \ldots$ This allows us to pick, for any $\lambda$-term $m$ any $i \geq \ell(m)$ and set unambiguously $\mathcal{V}[m]=\mathcal{V}_{i}[m]$. 
In this fashion we have defined a function $\mathcal{V}: \Lambda \rightarrow \mathcal{C}\left(X^{V}, X\right)$ such that the following conditions are satisfied:

(a) $(\forall x \in|X| \subseteq \Lambda, \rho \in \operatorname{Env}) \quad \mathcal{V}[x] \rho=x$, i.e., $\mathcal{V}[x] \in \mathcal{C}\left(X^{V}, X\right)$ is the constant function with value $x$.

(b) $(\forall v \in V, \rho \in \operatorname{Env}) \quad \mathcal{V}[v] \rho=\rho(v)$, i.e., on $V$ the function $\mathcal{V}$ agrees with the evaluation function, or projection, $V \rightarrow \mathcal{C}\left(X^{V}, X\right)$.

(c) $(\forall m, n \in \Lambda, \rho \in \mathrm{Env}) \quad \mathcal{V}[m n] \rho=(\mathcal{V}[m] \rho) \cdot(\mathcal{V}[n] \rho)$.

(d) $\mathcal{V}[\lambda v \cdot m](\rho)=j\left(\mathcal{V}[m] \circ \Phi_{\rho v}\right)$ for all $m \in \Lambda$ and all $(\rho, v) \in \operatorname{Env} \times V$.

This function $\mathcal{V}$ is called the semantic map of the model. It is uniquely determined by conditions (a,b,c,d).

The recursive definition of the semantic map is somewhat involved due to Definition 3.5. Therefore, it is very important for us to realize that the conditions of this definition are automatically satisfied as soon as we are dealing with the most prevalent type of functional domains, namely, the special ones as explained in Definition 3.1(iii) above.

Special Functional Domain Lemma. Every special functional domain $\left(X^{X}, j, p\right)$ in a concrete cartesian closed category with function spaces $\mathcal{C}$ (see 3.1(iii), A.11, A.13) is an environment model in $\mathcal{C}$. In particular, $\mathcal{V}[m] \in \mathcal{C}\left(X^{V}, X\right) \subseteq X^{\text {Env }}$ for all $m \in \Lambda$.

Proof. We have seen that for every morphism $F \in \mathcal{C}\left(X^{V}, X\right)$, the function

$$
\rho \mapsto F \circ \Phi_{\rho v}: X^{V} \rightarrow X^{X}
$$

is a $\mathcal{C}$-morphism. By induction hypothesis on $\mathcal{V}_{i}$, for all $m \in \Lambda_{i}$, the morphism $\mathcal{V}_{i}[m] \in \mathcal{C}\left(X^{V}, X\right)$ is well-defined. Thus

$$
\rho \mapsto \mathcal{V}[m] \circ \Phi_{\rho v}: X^{V} \rightarrow X^{X}
$$

is a $\mathcal{C}$-morphism by (*) above. Since $j: X^{X} \rightarrow X$ is a $\mathcal{C}$-morphism, the requirements of Definition 3.5 are satisfied with $[X \rightarrow X]=X^{X}$, and

$$
\mathcal{V}_{i+1}[\lambda v \cdot m]=\left(\rho \mapsto j\left(\mathcal{V}_{i}[m] \circ \Phi_{\rho v}\right)\right): X^{V} \rightarrow X
$$

is a $\mathcal{C}$-morphism. By induction on $i$, for all $i=1,2, \ldots$ we have $\mathcal{V}_{i}[m] \subseteq \mathcal{C}\left(X^{V}, X\right)$ for all $m \in \Lambda_{i}$.

We need the following result (cf. [4]):

Free Variable Lemma. If $([X \rightarrow X], j, p)$ is an environment model and $\mathcal{V}: \Lambda \rightarrow$ $X^{\text {Env }}$ is the semantic map, then

$$
(\forall \rho \in \operatorname{Env}) \quad \mathcal{V}[m] \rho=\mathcal{V}[m] \rho\{x / v\}
$$

if the variable $v$ is not free in the term $m$.

Proof. The proof is by induction on length. If $m$ is a constant $y \in X$, then the left and right sides of $(\star)$ both are $y$ and so the assertion holds. If $m \in V$, then $m \neq v$ by hypothesis; the left side of $(\star)$ is $\rho(m)$ the right side is $\rho\{x / v\}(m)=$ $\rho(m)$ by the definition of $\rho(x / v)$. Thus the assertion is true in this case. Now suppose that $(\star)$ is true for all $m \in \Lambda_{i}$. If $\mu \in \Lambda_{i+1}$ there are two cases: (1) $\mu=m n$ with $m, n \in \Lambda_{i}$ and $v$ free in neither $m$ nor $n$, or (2) $\mu=\lambda u . m$ 
with $m \in \Lambda_{i}$ and $v$ not free in $m$. In case (1), by the induction hypothesis, $\mathcal{V}[m] \rho=\mathcal{V}[m] \rho\{x / v\}$ and $\mathcal{V}[n] \rho=\mathcal{V}[n] \rho\{x / v\}$. Now (c) implies $\mathcal{V}[m n] \rho=$ $(\mathcal{V}[m] \rho) \cdot(\mathcal{V}[n] \rho)=(\mathcal{V}[m] \rho\{x / v\}) \cdot(\mathcal{V}[n] \rho\{x / v\})=\mathcal{V}[m n] \rho\{x / v\}$. Thus $(\star)$ holds for $\mu$ in place of $m$. In case (2), condition (d) implies $\mathcal{V}[\lambda u . m] \rho=j\left(\mathcal{V}[m] \circ \Phi_{\rho u}\right)$ and $\mathcal{V}[\lambda v \cdot m] \rho\{x / v\}=j\left(\mathcal{V}[m] \circ \Phi_{\rho\{x / v\} u}\right)$. Let $z \in X$. Then $\left(\mathcal{V}[m] \circ \Phi_{\rho u}\right)(z)=$ $V[m](\rho\{z / u\})$ and $\left(\mathcal{V}[m] \circ \Phi_{\rho\{x / v\} u}\right)(z)=V[m](\rho\{x / v\}\{z / u\})$. By (†) and by induction hypothesis, since $(\star)$ holds for $m \in \Lambda_{i}$, we have $\mathcal{V}[m](\rho\{z / v\}\{x / u\})=$ $\mathcal{V}[m](\rho\{z / u\})$. This yields the assertion.

\section{Environment models on functional domains}

In the following, we assume that the concrete cartesian closed category $\mathcal{C}$ has function spaces (see A.11, A.13) and we consider an environmental model $([X \rightarrow$ $X], j, p)$ in $\mathcal{C}$. In view of the existence of a grounding functor from $\mathcal{C}$ into $\mathcal{S E} \mathcal{T}$ we may regard the objects of $\mathcal{C}$ as sets (with additional structure). We shall therefore use a notation like $x \in X$ (in lieu of the more accurate $x \in|X|$ ); confusion is not likely to arise by such abuse of notation.

We recall $x \cdot y=p(x)(y)$ for all $x, y \in X$ and define $D_{\circ}=\{(x, y) \in X \times X$ : $p(x) \circ p(y) \in[X \rightarrow X]\}$. Then we have a partial binary operation $\circ: D_{\circ} \rightarrow X$ defined by $x \circ y=j(p(x) \circ p(y))$ for $(x, y) \in D_{\circ}$. From 3.2(i) we also recall the definition $e=j\left(\mathrm{id}_{X}\right)$.

For elements of $X^{\left(X^{V}\right)}$ we shall use the notation $\xi: X^{V} \rightarrow X$.

Now we define an algebraic structure on $X^{\left(X^{V}\right)}$ pointwise as follows: for $\xi \in X^{V}$ and $\eta \in X^{V}$ we set $(\xi \cdot \eta)(\rho)=\xi(\rho) \cdot \eta(\rho)$ for $\rho \in X^{V}$. Set

$$
D_{\circ}^{\prime}=\left\{(\xi, \eta) \in X^{\left(X^{V}\right)} \times X^{\left(X^{V}\right)}:\left(\forall \rho \in X^{V}\right) \quad(\xi(\rho), \eta(\rho)) \in D_{\circ}\right\} .
$$

We define the partial binary operation

$$
\circ: D_{\circ}^{\prime} \rightarrow X^{\left(X^{V}\right)} \quad \text { by } \quad(\xi \circ \eta)(\rho)=\xi(\rho) \circ \eta(\rho)
$$

for $\rho \in X^{V}$ and for all $(\xi, \eta) \in D_{0}^{\prime}$, and set $\epsilon(\rho)=e$ for all $\rho \in X^{V}$; thus $\epsilon$ is the constant function with value $\epsilon$.

We define

$$
p^{\prime}: X^{\left(X^{V}\right)} \rightarrow[X \rightarrow X]^{\left(X^{V}\right)} \quad \text { by } \quad p^{\prime}(f)=p \circ f
$$

for $f: X^{V} \rightarrow X$ and

$$
j^{\prime}:[X \rightarrow X]^{\left(X^{V}\right)} \rightarrow X^{\left(X^{V}\right)} \quad \text { by } \quad j^{\prime}(F)=j \circ F
$$

for $F: X^{V} \rightarrow[X \rightarrow X]$. Then $p^{\prime}$ and $j^{\prime}$ are $\mathcal{C}$-morphisms and $p^{\prime} \circ j^{\prime}=\mathrm{id}_{X^{\left(X^{V}\right)}}$. We recall that $[X \rightarrow X] \subseteq X^{X}$ and that there is a morphism

$$
\sigma:\left(X^{X}\right)^{\left(X^{V}\right)} \rightarrow\left(X^{\left(X^{V}\right)}\right)^{\left(X^{\left(x^{V}\right)}\right)} \quad \text { by } \quad \sigma(f)(F)(\rho)=f(\rho)(F(\rho))
$$

for $f: X^{V} \rightarrow X^{X}, F: X^{V} \rightarrow X$ and $\rho \in X^{V}$. (For a diagrammatic definition of $\sigma$ see the Appendix A.4(v). Thus we have a $\mathcal{C}$-inclusion

$$
i:[X \rightarrow X]^{\left(X^{V}\right)} \rightarrow\left(X^{\left(X^{V}\right)}\right)\left(X^{\left(x^{v}\right)}\right)
$$


We therefore identify $[X \rightarrow X]^{\left(X^{v}\right)}$ with a subobject of $\left(X^{\left(X^{v}\right)}\right)\left(X^{\left(x^{v}\right)}\right)$. Having done this, we note that $\left([X \rightarrow X]^{\left(X^{V}\right)}, j^{\prime}, p^{\prime}\right)$ is a functional domain on $X^{\left(X^{V}\right)}$ (see 3.1). Since the maps $j^{\prime}$ and $p^{\prime}$ are defined pointwise, we also note that $\left([X \rightarrow X]^{\text {Env }}, j^{\prime}, p^{\prime}\right)$ is a functional domain on $X^{\text {Env }}$. Notice that the identity map $\gamma: X^{\left(X^{V}\right)} \rightarrow X^{\text {Env }}$ is continuous; it is not open unless Env $=|X|^{V}$ is finite.

From the information in the preceding subsection we have a semantic map $\mathcal{V}: \Lambda \rightarrow \mathcal{C}\left(X^{V}, X\right) \subseteq|X|^{\text {Env }}$. Thus we may define

$$
T=\mathcal{V}(\Lambda) \subseteq \mathcal{C}\left(X^{V}, X\right) \subseteq|X|^{\text {Env }} .
$$

Note that $T$ is, at this point, just a set.

Lemma 3.6. If $([X \rightarrow X], j, p)$ is an environment model, then for each environment $\rho \in X^{V}$, the semantic map $\mathcal{V}: \Lambda \rightarrow X^{\left(X^{V}\right)}$ satisfies the following conditions:

(i) $(\forall m, n \in \Lambda) \quad \mathcal{V}[m n]=\mathcal{V}[m] \cdot \mathcal{V}[n]$,

(ii) $T \times T \subseteq D_{\circ}^{\prime}$ and $(\forall m, n \in \Lambda) \quad \mathcal{V}[\operatorname{compose}(m, n)]=\mathcal{V}[m] \circ \mathcal{V}[n]$,

(iii) $\mathcal{V}[\mathrm{I}]=\epsilon$.

(iv) The maps $:: T \times T \rightarrow T$ and $\circ: T \times T \rightarrow T$ are continuous with respect to each of the topologies induced by $X^{\left(X^{V}\right)}$ and $X^{\text {Env }}$.

Proof. (i) follows from condition (c) in the recursive definition of $\mathcal{V}$.

For (ii), we consider a variable $v$ which occurs freely in neither $m$ nor $n$ and, using the definition of an environment model in 3.5, we make the following calculation: $\mathcal{V}[\operatorname{compose}(m, n)] \rho=\mathcal{V}[\lambda v \cdot m(n v)] \rho=j(f)$ for any environment $\rho$ by $(\mathrm{d})$, where

$$
\begin{gathered}
f=x \mapsto \mathcal{V}[m(n v)] \rho\{x / v\}=x \mapsto p(\mathcal{V}[m] \rho\{x / v\})(\mathcal{V}[n v] \rho\{x / v\}) \text { by }(\mathrm{c}) \\
=x \mapsto p(\mathcal{V}[m] \rho\{x / v\})(p(\mathcal{V}[n] \rho\{x / v\})(\mathcal{V}[v] \rho\{x / v\}) \text { by }(\mathrm{c}) \\
=x \mapsto p(\mathcal{V}[m] \rho\{x / v\})(p(\mathcal{V}[n] \rho\{x / v\})(x)) \text { by }(\mathrm{b}) \\
=x \mapsto p(\mathcal{V}[m] \rho)(p(\mathcal{V}[n] \rho)(x)),
\end{gathered}
$$

the last equality following from the Free Variable Lemma and the fact that $v$ is free in neither $m$ nor in $n$. This shows that the element $f \in[X \rightarrow X]$ is the composition of the functions $p(\mathcal{V}[m] \rho)$ and (first) $p(\mathcal{V}[n] \rho)$. Thus $(\mathcal{V}[m] \rho, \mathcal{V}[n] \rho) \in D_{\circ}$ for all $\rho$ and so $(\mathcal{V}[m], \mathcal{V}[n]) \in D_{\circ}^{\prime}$. Hence $T \times T \subseteq D_{\circ}^{\prime}$ as asserted. Thus (ii) is proved in its entirety. $\rho$, where

For (iii), we again calculate: $\mathcal{V}[\mathrm{I}] \rho=\mathcal{V}[\lambda v \cdot v] \rho=j(f)$ for any environment

$$
\begin{aligned}
f=x^{\prime} \mapsto \mathcal{V}[v] \rho\left\{x^{\prime} / v\right\} & =x^{\prime} \mapsto \rho\left\{x^{\prime} / v\right\}\left(x^{\prime}\right) \\
& =x^{\prime} \mapsto x^{\prime} .
\end{aligned}
$$

This proves the claim.

The assertion (iv) follows since the operations $\cdot$ and $\circ$ are defined pointwise.

One comment regarding (ii) is in order. The relation $T \times T \subseteq D_{\circ}^{\prime}$ is perhaps surprising; it has the flavor of getting something for nothing. However, what is used here is the information coded into the Definition 3.5 of an environment model. 
Lemma 3.7. If $m$ and $n$ are $(\alpha),(\beta)$ or $(\eta)$-equivalent, then $\mathcal{V}[m]=\mathcal{V}[n]$.

Proof. We record

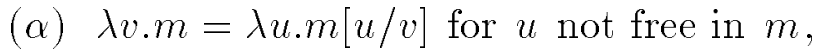

( $\beta)(\lambda v \cdot m) q=m[q / v]$, and

( $\eta) \lambda v \cdot(m v)=m$ for $v$ not free in $m$.

Proof for $(\alpha): \mathcal{V}[\lambda v \cdot m] \rho=j\left(\mathcal{V}[m] \circ \Phi_{\rho v}\right)$ on the one hand and $\mathcal{V}[\lambda u \cdot m[u / v]] \rho=$ $j\left(\mathcal{V}[m[u / v]] \circ \Phi_{\rho u}\right)$ on the other. In order for these to be equal it is necessary and sufficient that $\mathcal{V}[m] \rho\{x / v\}=\left(\mathcal{V}[m] \circ \Phi_{\rho v}\right)(x)=\left(\mathcal{V}[m[u / v]] \circ \Phi_{\rho u}\right)(x)=$ $\mathcal{V}[m[u / v]] \rho\{x / u\}$. This claim is proved by induction on the length of $m$. Indeed, if $m$ is constant, then $\mathcal{V}[m[u / v]] \rho=\mathcal{V}[m] \rho$ for all environments $\rho$. If $m=w \in V$, we have $m[u / v]=m$ if $w \neq v$, so $\mathcal{V}[m[u / v]] \rho=\mathcal{V}[m] \rho$ for all environments $\rho$ in this case. Alternatively, if $w=v$, then $m[u / v]=u$, and so $\mathcal{V}[m[u / v]] \rho\{x / v\}=x=\mathcal{V}[u] \rho\{x / u\}$ for all environments $\rho$. Hence the result also holds in case $m$ is a variable, and this concludes the proof for the cases when $\ell(m)=1$. If the result holds for all terms $m$ with $\ell(m)<i$, and $m=n p$ is an application, then the result holds for $m$ by the inductive hypothesis and the fact that $\mathcal{V}$ is an application-homomorphism (3.6(ii)).

Finally, suppose $m=\lambda w \cdot n$ is itself an abstraction. Then the definition of $\mathcal{V}[m]=\mathcal{V}[\lambda w \cdot n]$ yields

$$
\mathcal{V}[m] \rho\{x / v\}=\mathcal{V}[\lambda w \cdot n] \rho\{x / v\}=j(\mathcal{V}[n] \rho\{x / v\}\{x / w\})
$$

while

$$
\mathcal{V}[m[u / v]] \rho\{x / u\}=\mathcal{V}[(\lambda w \cdot n)[u / v]] \rho\{x / u\}=j(\mathcal{V}[n[u / v]] \rho\{x / u\}\{x / w\}) .
$$

By the inductive hypothesis, $\mathcal{V}[n] \rho\{x / v\}\{x / w\}=\mathcal{V}[n[u / v]] \rho\{x / u\}\{x / w\}$ since these terms have shorter length, and this proves the result for $(\alpha)$.

Proof for $(\beta)$ : We have

$$
\begin{aligned}
\mathcal{V}[(\lambda v \cdot m) q] \rho & =(\mathcal{V}[\lambda v \cdot m] \rho) \cdot(\mathcal{V}[q] \rho) \\
& =\left(\mathcal{V}[m] \circ \Phi_{\rho v}\right)(\mathcal{V}[q] \rho) \\
& =\mathcal{V}[m] \rho\{(\mathcal{V}[q] \rho) / v\},
\end{aligned}
$$

and we claim that this equals $\mathcal{V}[m[q / v]] \rho$. Again this is proved by induction on the length of $m$, and the arguments are straightforward and similar to the case of the $(\alpha)$ rule.

Proof of $(\eta)$ : We compute $\mathcal{V}[\lambda v \cdot(m v)] \rho=j\left(\mathcal{V}[m v] \circ \Phi_{\rho}\right)$. Now for $x \in X$ we have $y \stackrel{\text { def }}{=}\left(\mathcal{V}[m v] \circ \Phi_{\rho v}\right)(x)=\mathcal{V}[m v] \rho\{x / v\}=(\mathcal{V}[m] \rho\{x / v\}) \cdot(\mathcal{V}[v] \rho\{x / v\})$. Ву the Free Variable Lemma, $\mathcal{V}[m] \rho\{x / v\}=\mathcal{V}[m] \rho$ if $v$ is not free in $m$. Further $\mathcal{V}[v] \rho\{x / v\}=\rho\{x / v\}(v)=x$ by the definitions of $\mathcal{V}$ and $\rho\{x / v\}$. Hence $y=$ $\mathcal{V}[m] \rho \cdot x$. Thus $\mathcal{V}[m v] \circ \Phi_{\rho}=p(\mathcal{V}[m] \rho)$, whence $\mathcal{V}[\lambda v \cdot(m v)] \rho=j p(\mathcal{V}[m] \rho)=$ $\mathcal{V}[m] \rho$, i.e., $\mathcal{V}[\lambda v \cdot(m v]=\mathcal{V}[m]$ as asserted.

Lemma 3.8. (i) $(T, \circ)$ is a monoid. The set $M \stackrel{\text { def }}{=} p^{\prime}(T) \subseteq\left|[X \rightarrow X]^{\left(X^{V}\right)}\right|$ is a submonoid of $\left|\left(X^{V}\right)^{\left(X^{V}\right)}\right|=\mathcal{C}\left(X^{V}, X^{V}\right)$. If $M$ is a $\mathcal{C}$-subobject of $[X \rightarrow X]$, then both $\left(M,[X \rightarrow X]^{\left(X^{V}\right)}, j^{\prime}, p^{\prime}\right)$ and $\left(M,[X \rightarrow X]^{\text {Env }}, j^{\prime}, p^{\prime}\right)$ are functional monoids (see 3.1(ii)).

(ii) $j$ maps $M$ isomorphically onto a submonoid $S$ of $T$ whose identity is $\epsilon$, $\epsilon(\rho)=e$, with $e=j\left(\mathrm{id}_{X}\right)$. Also, $T$ is an inflation of $S$ with respect to $\xi \mapsto \epsilon \circ \xi$. 
(iii) T acts $\epsilon$-effectively on $\left|X^{\left(X^{V}\right)}\right|$.

Proof. (i) $T$ consists exactly of all $\mathcal{V}[m]: X^{V} \rightarrow X$ with $m \in \Lambda$. By 3.6(ii) and (iii) these elements form a submonoid of $\left(X^{\left(X^{V}\right)}\right)\left(X^{\left(x^{V}\right)}\right)$ contained in $[X \rightarrow$ $X]^{\left(X^{V}\right)}$. If $\xi, \eta$ and $\zeta$ are elements of $T$ with $x_{\rho}=\mathcal{V}[m] \rho, y_{\rho}=\mathcal{V}[n] \rho$, and $z_{\rho}=\mathcal{V}[r] \rho$, then

$$
((\xi \circ \eta) \cdot \zeta)(\rho)=\mathcal{V}[\operatorname{compose}(m, n) r](\rho)=\mathcal{V}[m(n r)](\rho) \quad \text { for } \quad \rho \in X^{V}
$$

and thus $(\xi \circ \eta) \cdot \zeta \in T$ by 3.4 and $3.7(\beta)$. Again using 3.6, this last expression is equal to $(\xi \circ \eta) \cdot \zeta$. Thus $p(\xi \circ \eta)=p(\xi) \circ p(\eta)$. Hence $M$ is closed under composition in $\left(X^{V}\right)^{\left(X^{V}\right)}$ and contains the identity $p(\epsilon)$. Also, $p^{\prime} \mid T:(T, 0) \rightarrow(M, 0)$ is a monoid morphism. The remainder is clear from the definitions.

(ii) $j$ is injective and transports composition in $M$ to the o operation in $T$. The image of the identity of $M$ is $\epsilon$. The definition of the multiplication $\circ$ directly implies that $T$ is an inflation of $S$ via multiplication with $\epsilon$.

(iii) Suppose that $\xi, \eta: X^{V} \rightarrow X$ are in $T$ with $\xi=\mathcal{V}[\mathrm{m}]$ and $\eta=\mathcal{V}[n]$, and that $\zeta: X^{V} \rightarrow X$. Then $\xi \cdot(\eta \cdot \zeta)(\rho)=p^{\prime}(\xi)\left(p^{\prime}(\eta)(\zeta)\right)(\rho)=\{p(\mathcal{V}[m] \rho) \circ p(\mathcal{V}[n] \rho)\} \cdot \zeta(\rho)$ for $\rho \in X^{V}$ on the one hand, and $(\xi \circ \eta) \cdot \zeta(\rho)=p^{\prime}(\xi \circ \eta)(\zeta)(\rho)=\{p(\mathcal{V}[m] \rho) \circ$ $p \mathcal{V}[n] \rho)\} \cdot \zeta(\rho)$ on the other, and this shows that we have an action. The relation $\epsilon \cdot \xi=\xi$ is readily verified. Finally suppose that $\xi \cdot \zeta=\eta \cdot \zeta$. Then for all $\rho \in X^{V}$ we have $\xi(\rho) \cdot \zeta(\rho)=\eta(\rho) \cdot \zeta(\rho)$. From 3.2(ii) we have $\epsilon \circ \xi(\rho)=e \circ \eta(\rho)$. Thus $\epsilon \circ \xi=\epsilon \circ \eta$.

Environment models in standard topological categories

Now we assume that $\mathcal{C}$ is a standard topological category (see 3.2).

Proposition 3.9. Suppose that $([X \rightarrow X], j, p)$ is an environment model in a cartesian closed category with function spaces. Set $T=\mathcal{V}[\Lambda] \subseteq \mathcal{C}\left(X^{V}, X\right)=\left|X^{\left(X^{V}\right)}\right|$ and endow this set with either the topology induced from that of $X^{\left(X^{V}\right)}$ or that of $X^{\text {Env }}$. Then, in both cases, $(T, T)$ is a strict functional algebra according to Definition 2.10 .

Proof. By Lemma 3.7 conditions (i) and (ii) of Definition 2.1 are satisfied. Recall the paradoxical combinator $\mathbf{Y}=\lambda f .(\lambda v \cdot f(v v))(\lambda v \cdot f(v v))$; then $\mathbf{Y} m$ is $\eta$-equivalent to $m(\mathbf{Y} m)$. Hence

$$
\mathcal{V}[\mathbf{Y}] \cdot \mathcal{V}[m]=\mathcal{V}[\mathbf{Y} m]=\mathcal{V}[m(\mathbf{Y} m)]=\mathcal{V}[m] \cdot(\mathcal{V}[\mathbf{Y}] \cdot \mathcal{V}[m])
$$

by 3.6(i) and $3.8(\eta)$. Thus hypothesis (iii) of 2.1 is satisfied in $(T, T)$ with $y=$ $\mathcal{V}[\mathbf{Y}] \in T$. Next recall $\mathbf{K} \in \Lambda, \mathbf{K}=\lambda u$. $(\lambda v \cdot u)$. Then $\mathbf{K} m$ is $\eta$-equivalent to $\lambda v \cdot m$ for all $m \in \Lambda$. Thus $(\mathbf{K} m) n$ is equivalent to $(\lambda v \cdot m) n$ which is equivalent $m$. Hence, by $3.8(\eta)$, we have $(V[\mathbf{K}] \cdot \mathcal{V}[m]) \mathcal{V}[n]=\mathcal{V}[(\mathbf{K} m) n]=V[m]$, and so $k=\mathcal{V}[\mathbf{K}] \in T$ satisfies $\left(7^{\prime}\right)$ of 3.6 in $T$. It follows that $K: T \rightarrow T, K(\xi)=k \cdot \xi$ satisfies $(7)$ of Definition 2.1. This completes the proof that $(T, T)$ is a strict functional algebra in the sense of 2.1 .

Proposition 3.10. Suppose that $([X \rightarrow X], j, p)$ is an environment model in a standard topological category. Set $T=\mathcal{V}[\Lambda] \subseteq \mathcal{C}\left(X^{V}, X\right)=\left|X^{\left(X^{V}\right)}\right|$. Denote with $\bar{T}$ the closure of $T$ in $X^{\left(X^{V}\right)}$ and with $\widetilde{T}$ the closures of $T$ in $X^{\text {Env }}$, given 
the respective induced topologies. Assume that $[X \rightarrow X]$ is closed in $X^{X}$. Then $\bar{T} \times \bar{T} \subseteq \widetilde{T} \times \widetilde{T} \subseteq D_{\circ}^{\prime}$ and both $(\bar{T}, \bar{T})$ and $(\widetilde{T}, \widetilde{T})$ are strict functional algebras.

Proof. We claim that $D_{\circ} \subseteq X \times X$ is closed: the function $\gamma=[(x, y) \mapsto$ $p(x) \circ p(y)]: X \times X \rightarrow X^{X}$ is continuous since $p$ is continuous and $\mathcal{C}$ is cartesian closed. We have $D_{\circ}=\gamma^{-1}[X \rightarrow X]$. Since $[X \rightarrow X]$ is closed in $X^{X}$ by hypothesis, the claim

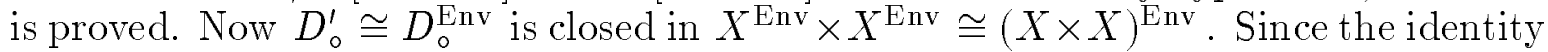
$\gamma: X^{\left(X^{V}\right)} \rightarrow X^{\text {Env }}$ is continuous, $D_{\mathrm{o}}^{\prime}$ is also closed in $X^{\left(X^{V}\right)} \times X^{\left(X^{V}\right)} \cong(X \times X)^{\left(X^{V}\right)}$. From the continuity of $\gamma$ we also obtain that $\bar{T} \subseteq \widetilde{T}$. It follows from 3.6(ii) that $\bar{T} \times \bar{T} \subseteq \widetilde{T} \times \widetilde{T} \subseteq D_{\circ}^{\prime}$ whence $(\bar{T}, \circ)$ and $(\widetilde{T}, \circ)$ are binary algebras with identity $\epsilon$ and a continuous multiplication $O$ in each case; since $T$ is a dense submonoid by 3.9, we have that $(\bar{T}, 0)$ and $(\widetilde{T}, \circ)$ are monoids. Also the fact that $T$ is closed with respect to the continuous binary operations $: X^{\left(X^{V}\right)} \times X^{\left(X^{V}\right)} \rightarrow X^{\left(X^{V}\right)}$ and $\cdot: X^{\text {Env }} \times X^{\text {Env }} \rightarrow X^{\text {Env }}$ (identical as functions) implies that the restrictions $\cdots: \bar{T} \times \bar{T} \rightarrow \bar{T}$ and $\cdots: \widetilde{T} \times \widetilde{T} \rightarrow \widetilde{T}$ are well-defined. All equations involving the binary operations $o$ and $\cdot$ and the elements $k$ and $y$ extend by continuity. The $\epsilon$-effectivity of the action of $\bar{T}$ on $\bar{T}$ is directly established as in the last lines of the proof of 3.8(iii).

Theorem 3.11. Assume the following hypotheses:

(a) $\mathcal{C}$ is a standard topological category.

(b) $([X \rightarrow X], j, p)$ is an environment model on a compact Hausdorff space $X$. Then the semantic map $\mathcal{V}: \Lambda \rightarrow|X|^{\text {Env }}$ is constant.

Proof. Since $X$ is compact, then $[X \rightarrow X]=p(X)$ is compact and hence closed in $X^{X}$ as $X$ is Hausdorff. Then by Proposition 3.10 we know that $(\bar{T}, \bar{T})$ with $T=\mathcal{V}[\Lambda]$ is a functional algebra. Since $X$ is compact, Tychonov's Theorem implies $X^{\text {Env }}$ is compact and thus $\widetilde{T} \subseteq X^{\text {Env }}$ is compact, too. Thus $(\widetilde{T}, \widetilde{T})$ is a compact Hausdorff functional algebra. By Theorem 2.7, it is degenerate. Hence $T$ is singleton and this is the assertion.

For a better understanding of Theorem 3.11 it should be recalled from the Special Functional Domain Lemma that every special functional domain $\left(X^{X}, j, p\right)$ in $\mathcal{C}$ (see $3.1($ iii)) is an environment model.

Corollary 3.12. In the category of Hausdorff $k$-spaces any compact environmental model (in particular every compact special functional domain) is trivial in the sense that its semantic map is constant.

\section{Combinatory Models}

In this section we fix a cartesian closed category with function spaces (see A.11 and A.13).

The following definition of a combinatory model is adjusted from [6] in order to allow for models to be implemented in a suitable standard topological category $\mathcal{C}$ :

Definition 4.1. Let $\mathcal{C}$ denote a concrete cartesian closed category with function spaces.

(i) A combinatory $\mathcal{C}$-algebra is a $\mathcal{C}$-object $X$ together with a binary $\mathcal{C}$-operation $\cdot: X \times X \rightarrow X$ such that there are elements $K, S \in X$ satisfying

(a) $\left(\forall x_{0}, x_{1} \in X\right)\left(K \cdot x_{0}\right) \cdot x_{1}=x_{0}$,

(b) $\left(\forall x_{0}, x_{1}, x_{2} \in X\right)\left(\left(S \cdot x_{0}\right) \cdot x_{1}\right) \cdot x_{2}=\left(x_{0} \cdot x_{2}\right) \cdot\left(x_{1} \cdot x_{2}\right)$. 
(ii) A combinatory $\mathcal{C}$-model is a combinatory $\mathcal{C}$-algebra $(X, \cdot)$ which also has a distinguished element $\epsilon \in X$ satisfying, for all $x_{0}, x_{1} \in X$ :

(c) $\left(\epsilon \cdot x_{0}\right) \cdot x_{1}=x_{0} \cdot x_{1}$,

(d) if $(\forall x \in X) x_{0} \cdot x=x_{1} \cdot x$, then $\epsilon \cdot x_{0}=\epsilon \cdot x_{1}$, and

(e) $\epsilon \cdot \epsilon=\epsilon$.

The combinatory $\mathcal{C}$-model $(X, \cdot)$ is extensional if $\epsilon$ acts as an identity on $X$.

On the free binary algebra $A$ generated by $K$ and $S$ and a set $V$ of variables one defines $I=S(K K)$ and, for each variable $x$ an operation $P \mapsto \lambda^{*} x . P$ by induction via

1) $(\forall x \in V) \quad \lambda^{*} x . x=I$,

2) $(\forall x \in V) \quad \lambda^{*} x \cdot P=K P$,

3) $(\forall x \in V) \quad \lambda^{*} x \cdot P Q=S\left(\left(\lambda^{*} x . P\right)\left(\lambda^{*} x \cdot Q\right)\right)$.

In particular, we define

$$
\begin{aligned}
W & =\lambda^{*} x . f(x x)=S\left(\left(\lambda^{*} x . f\right)\left(\lambda^{*} x . x x\right)\right) \\
& =S\left[(K f)\left(S\left(\lambda^{*} x \cdot x\right)\left(\lambda^{*} x \cdot x\right)\right)\right]=S[(K f)((S(I I))],
\end{aligned}
$$

and, accordingly,

$$
\begin{aligned}
\lambda^{*} f . W & =\left(\lambda^{*} f . S\right)\left[\lambda^{*} f .(K f)(S(I I))\right] \\
& =(K S)\left[\left(\lambda^{*} f \cdot(K f)\right)(K(S(I I)))\right] \\
& =(K S)[((K K) I)(K(S(I I)))] .
\end{aligned}
$$

Finally we define

$$
\begin{aligned}
Y & =\lambda^{*} f . W W=S\left(\left(\lambda^{*} f . W\right)\left(\lambda^{*} f . W\right)\right) \\
& =S(\{(K S)[((K K) I)(K(S(I I)))]\}\{(K S)[((K K) I)(K(S(I I)))]\}) .
\end{aligned}
$$

In the same spirit, we introduce a binary operation $\circ: X \times X \rightarrow X$ via the $\lambda^{*}$-abstraction and Definition 3.3 as follows:

$$
\begin{aligned}
P \circ Q & =\lambda^{*} x \cdot P(Q x)=S\left(\left(\lambda^{*} x \cdot P\right)\left(\lambda^{*} x \cdot Q x\right)\right) \\
& =S((K P)[S\{(K Q) I\}]) .
\end{aligned}
$$

Then referring to $[2], p p .127,128,147 \mathrm{ff}$. for the details, we obtain

Proposition 4.2. In any combinatory $\mathcal{C}$-algebra we have

\section{Defining}

(f) $(\forall x \in X) \quad x \cdot(Y \cdot x)=Y \cdot x$.

$$
\text { ०: } X \times X \rightarrow X \quad \text { by } \quad x_{1} \circ x_{2}=S \cdot\left(\left(K \cdot x_{1}\right) \cdot\left[S \cdot\left\{\left(K \cdot x_{2}\right) \cdot I\right\}\right]\right)
$$

we obtain
(g) $\left(\forall x_{1}, x_{2}, x_{3} \in X\right) \quad\left(x_{1} \circ x_{2}\right) \cdot x_{3}=x_{1} \cdot\left(x_{2} \cdot x_{3}\right)$,
(h) $\left(\forall x_{1}, x_{2}, x_{3} \in X\right)\left(x_{1} \circ x_{2}\right) \circ x_{3}=x_{1} \circ\left(x_{2} \circ x_{3}\right)$.

If $X$ is an extensional $\mathcal{C}$-model, then $\left(\epsilon \circ x_{1}\right) \cdot x_{2}=\epsilon \cdot\left(x_{1} \cdot x_{2}\right)=x_{1} \cdot x_{2}$. Thus $\epsilon \cdot\left(\epsilon \circ x_{1}\right)=\epsilon \cdot x_{1}$ by $(\mathbf{d}, \mathbf{e})$. Since $X$ is extensional we conclude $\epsilon \circ x=x$ for all $x$. Likewise $\left(x_{1} \circ \epsilon\right) \cdot x_{2}=x_{1} \cdot\left(\epsilon \cdot x_{2}\right)=x_{1} \cdot x_{2}$ since $\mathcal{C}$ is extensional. Again we conclude $x \circ \epsilon=x$ for all $x$. Thus we note 
Proposition 4.3. In any extensional $\mathcal{C}$-model $X$ the object $X$ is a $\mathcal{C}$-monoid with respect to $\circ$ and $\epsilon$ as identity and $\cdot: X \times X \rightarrow X$ is an effective $\mathcal{C}$-action on $X$.

For the concept of a $\mathcal{C}$-action see A.7(iv)

Combinatory models in standard topological categories

Now we assume that $\mathcal{C}$ is a standard topological category (see 3.2). From 4.1(a), 4.2(f,g,h), and Proposition 4.3 we obtain at once:

Proposition 4.4. If $(X, \cdot)$ is an extensional combinatory model in a standard topological category, then $(X, X)$ is a strict functional algebra with respect to the $\mathcal{C}$-monoid $(X, \circ)$ with identity $\epsilon$ (see Definitions $2.1,2.10)$ and with respect to the elements $K$ and $Y$.

We say that a combinatory model in a standard topological category is compact if the underlying topological space $X$ is compact Hausdorff. A singleton combinatory model is called degenerate.

From Theorem 2.7 we now obtain at once the following result:

Theorem 4.5. Any compact extensional combinatorial model in a standard topological category is degenerate.

Corollary 4.6. Any compact extensional combinatorial model in the category of Hausdorff $k$-spaces is degenerate.

\section{Conclusion}

We have derived some results about compact Hausdorff monoids and shown how they imply the degeneracy of certain models of the untyped lambda calculus. As we commented at the end of Section 2, the bicyclic semigroup $B(k, y)$ plays a fundamental role in this setting. Its only homomorphic images are copies of itself, or cyclic groups, and compact semigroups cannot contain a copy of the former. Hence, in a compact Hausdorff semigroup which either is a functional monoid or a combinatory algebra, the elements $k$ and $y$ must be inverses of one another, and this forces the model to degenerate.

The main results are Theorem 2.7, 3.11, 3.12 and 4.5, and they are related as follows: Theorem 2.7 is a basic result on topological spaces and compact topological semigroup actions, while Theorems 3.11 and 4.5 and Corollary 3.12 deal with compact structures in a given standard topological category. All of these results are degeneracy theorems and the last two directly derive from 2.7. The generality of our definitions $3.1,3.5$, and 4.1 does not appear to allow us to deduce either of the Theorems 3.11 or 4.5 from the other. If we assume that the functional domain $D=([X \rightarrow X], p, j)$ is special (3.1(iii)), i.e. that $[X \rightarrow X]=X^{X}$, then $D$ is an environment model by the Special Functional Domain Lemma (following Definition 3.5). Also in this case a proof of Theorem 4.5 can be derived from Theorem 3.11: We may create from $D$ an extensional combinatory model by defining $x_{1} \cdot x_{2}=p\left(x_{1}\right)\left(x_{2}\right)$ and defining $\left.K=j\left(j \circ \kappa_{X}^{X}\right)\right)$ and $S=j\left(j \circ j^{j} \circ \sigma_{X}^{X}{ }^{X} \circ p^{X} \circ p\right)$.

\section{A. Appendix: Concrete cartesian closed categories}

In this section we provide the necessary background on cartesian closed categories. In a category $\mathcal{C}$ with finite products we shall abbreviate $A \times B$ by $A B$. The cartesian product in a category with finite products is associative (and commutative). 
We note that this implies there is a natural isomorphism $A(B C) \cong(A B) C$ (respectively $A B \cong B A$ ) and that in general the use of these isomorphisms involves the consideration of coherence in the sense of MACLANE. However, with the particular case of cartesian products in a category with finite products we will not encounter any difficulties if we simply write $(A B) C=A(B C)$ and in fact omit parentheses as is customary in group and semigroup theory.

Definition A.1. We say that a category $\mathcal{C}$ is cartesian closed if it has finite products and the functor $X \mapsto X A$ is a left adjoint for every $A$. Its right adjoint is denoted $X \mapsto X^{A}$.

Notation: if $A$ and $B$ are objects in $\mathcal{C}$, then $B^{A}$ is the object in $\mathcal{C}$ just defined. If $J$ is a set and $\mathcal{C}$ has products, then $B^{J}$ is the usual product object of $\mathcal{C}$. Confusion could conceivably arise if $\mathcal{C}=\mathcal{S E T}$. In that case, however, the two notations agree.

By interpreting this adjunction in terms of the back adjunction, this definition is equivalent to the following statement:

Remark A.2. For each object $A$ there is a natural transformation (in $B$ ) $\mathrm{ap}_{B}^{A}: B^{A} A \rightarrow B$ such that for any morphism $b: X A \rightarrow B$ there is a unique

$$
b^{\prime}: X \rightarrow B^{A}
$$

such that $\operatorname{ap}_{B}^{A} \circ\left(b^{\prime} A\right)=b$.

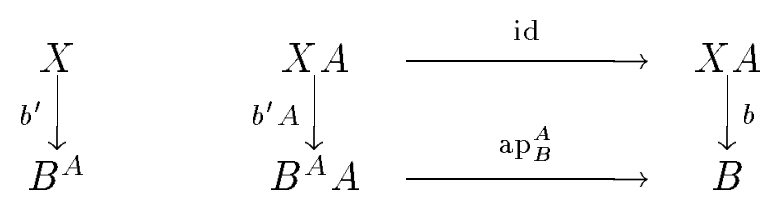

The uniqueness immediately yields the following egalization principle:

Remark A.3. Suppose that $\alpha, \beta: X \rightarrow B^{A}$ are two morphisms such that

$$
\operatorname{ap}_{B}^{A} \circ(\alpha A)=\operatorname{ap}_{B}^{A} \circ(\beta A) .
$$

Then $\alpha=\beta$.
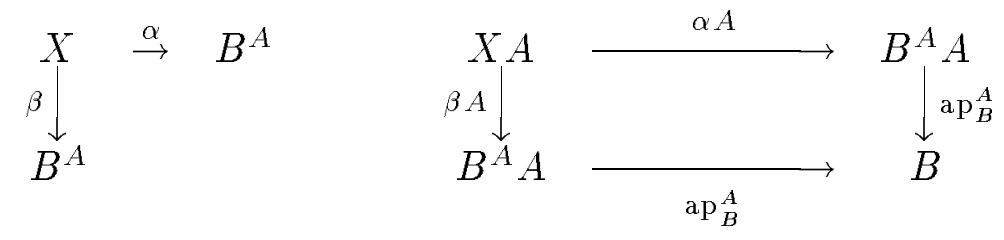

The principle allows some immediate canonical constructions.

Firstly, taking $X=B$ and $b=\operatorname{pr}_{1}$ in the universal property in A.2 we obtain a unique natural morphism $\kappa_{B}^{A}: B \rightarrow B^{A}$ such that the following diagram commutes

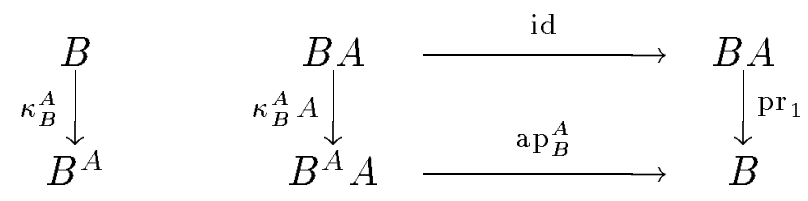


(In the category of sets, $\kappa_{B}^{A}$ is the function which associates with an $x \in B$ the constant function $\kappa_{x}: A \rightarrow B$ with value $x$.)

Secondly, we consider $C^{B} B^{A}$ and get a morphism

$$
\mu=\left(C^{B} B^{A} A \stackrel{C^{B} \mathrm{ap}_{B}^{A}}{\longrightarrow} C^{B} B \longrightarrow \mathrm{ap}_{C}^{B} \longrightarrow C .\right)
$$

The universal property above gives us a unique morphism

$$
{ }^{\circ} C B A: C^{B} B^{A} \rightarrow C^{A}
$$

such that $\operatorname{ap}_{C}^{A} \circ\left({ }^{\circ} B A A\right)=\mu$. In diagrams:
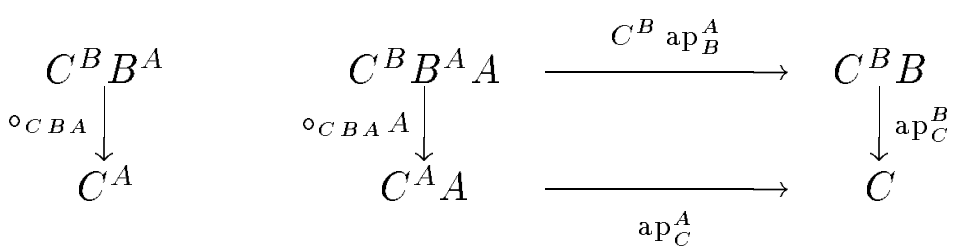

Thirdly, every morphism $\phi: A_{1} \rightarrow A_{2}$ induces functorially a morphism $B^{\phi}: B^{A_{2}} \rightarrow A^{A_{1}}$ via the following diagram:

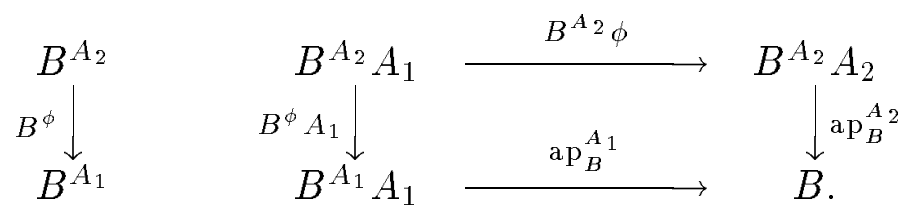

It is readily checked that $\phi \mapsto B^{\phi}: \mathcal{C} \rightarrow \mathcal{C}^{\text {op }}$ is a functor form $\mathcal{C}$ into its opposite category, i.e., that it is a contravariant functor.

Fourthly, every morphism $\phi: B_{1} \rightarrow B_{2}$ induces functorially a morphism $\phi^{A}: B_{1}^{A} \rightarrow B_{2}^{A}$ via the diagram

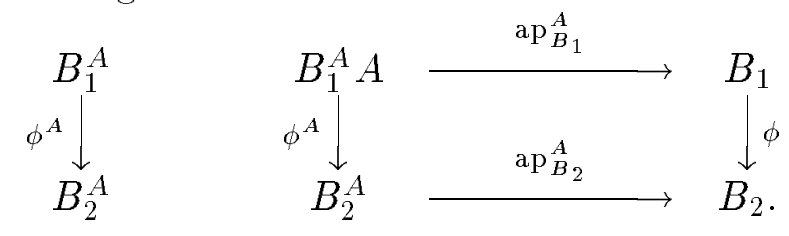

It is even easier to check that $\phi \mapsto \phi^{A}: \mathcal{C} \rightarrow \mathcal{C}$ is a functor.

Fifthly, as an exercise, we note a morphism $\delta: C^{A} B^{A} \rightarrow(C B)^{A}$ : firstly, for an object $A$ in a category with products let $\Delta_{A}: A \rightarrow A A$ be the diagonal morphism uniquely defined by $\operatorname{pr}_{j} \Delta=\mathrm{id}_{A}$ for $j=1,2$. Ten let $\nu:\left(C^{A} B^{A}\right) A \rightarrow\left(C^{A} A\right)\left(B^{A} A\right)$ be defined as the composition

$$
\left(C^{A} B^{A}\right) A \stackrel{\left(C^{A} B^{A}\right) \Delta_{A}}{\longrightarrow}\left(C^{A} B^{A}\right) A A \stackrel{\cong}{\cong}\left(C^{A} A\right)\left(B^{A} A\right) .
$$

Then we get $\delta: C^{A} B^{A} \rightarrow(C B)^{A}$ via

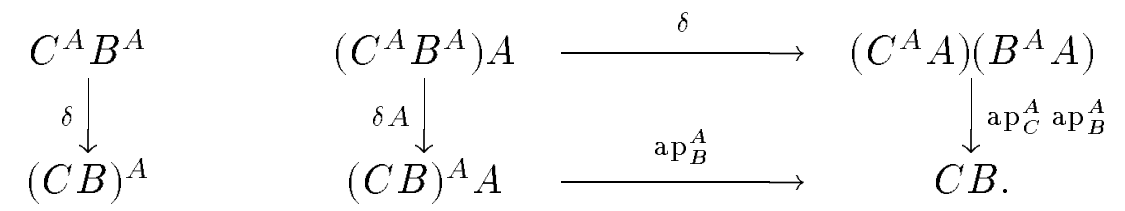


Next we define $\delta^{\prime}:(C B)^{A} \rightarrow\left(C^{A}\right)\left(B^{A}\right)$, using the universal property of the product, by $\operatorname{pr}_{j} \circ \delta^{\prime}=\left(\mathrm{pr}_{j}\right)^{A}, j=1,2$. It is an exercise to verify $\delta^{\prime}=\delta^{-1}$. Thus $\delta$ is an isomorphism.

Then the following diagrams define a natural morphism

$$
\sigma_{C}^{B A}:\left(C^{B}\right)^{A} \rightarrow\left(C^{A}\right)^{\left(B^{A}\right)}:
$$

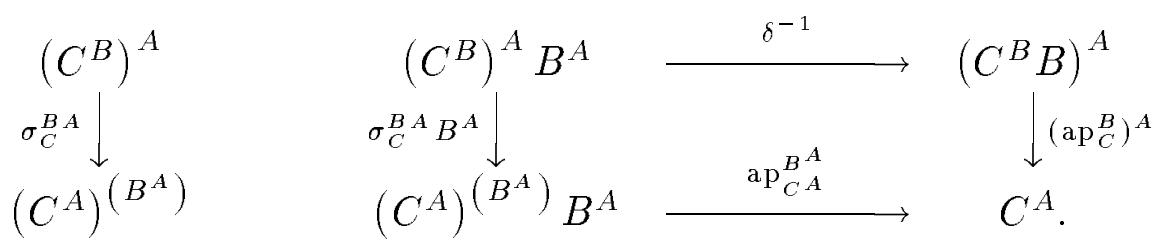

Definition A.4. (i) The morphism $\kappa_{B}^{A}$ is called the constant-picker,

(ii) the morphism ${ }^{\circ} C B A$ is called composition,

(iii) the assignment $\phi \mapsto B^{\phi}$ is called the contravariant power object functor,

and

(iv) the assignment $\phi \mapsto \phi^{A}$ is called the the covariant power object functor.

(v) The morphism $\sigma_{C}^{B A}$ is called the $S$-morphism (for simple reference only).

The $S$-morphism $\sigma$ corresponds to the $\mathbf{S}$ combinator in combinatory algebras (see 4.1). We use it e.g. in the discussions leading up to Lemma 3.6.

Proposition A.5. Composition is associative. Specifically, the following diagram is commutative:

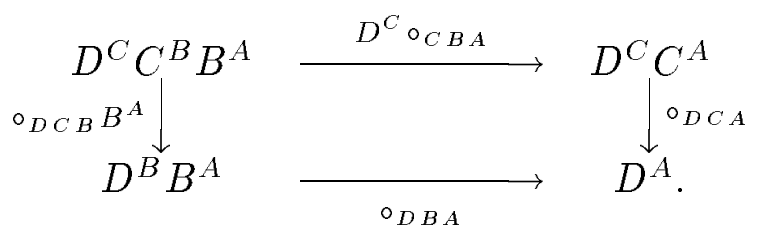

Proof. In order to prove the commuting of diagram (1) we use Remark 3 and set

$$
\alpha=\circ_{D B A} \circ\left({ }_{D C B} B^{A}\right) \text { and } \beta={ }^{\circ}{ }_{D C A} \circ\left(D^{C}{ }^{\circ}{ }_{C B A}\right) \text {. }
$$

We must show

$$
\operatorname{ap}_{D}^{A} \circ \alpha=\operatorname{ap}_{D}^{A} \circ \beta
$$

For this purpose we consider the following diagram:

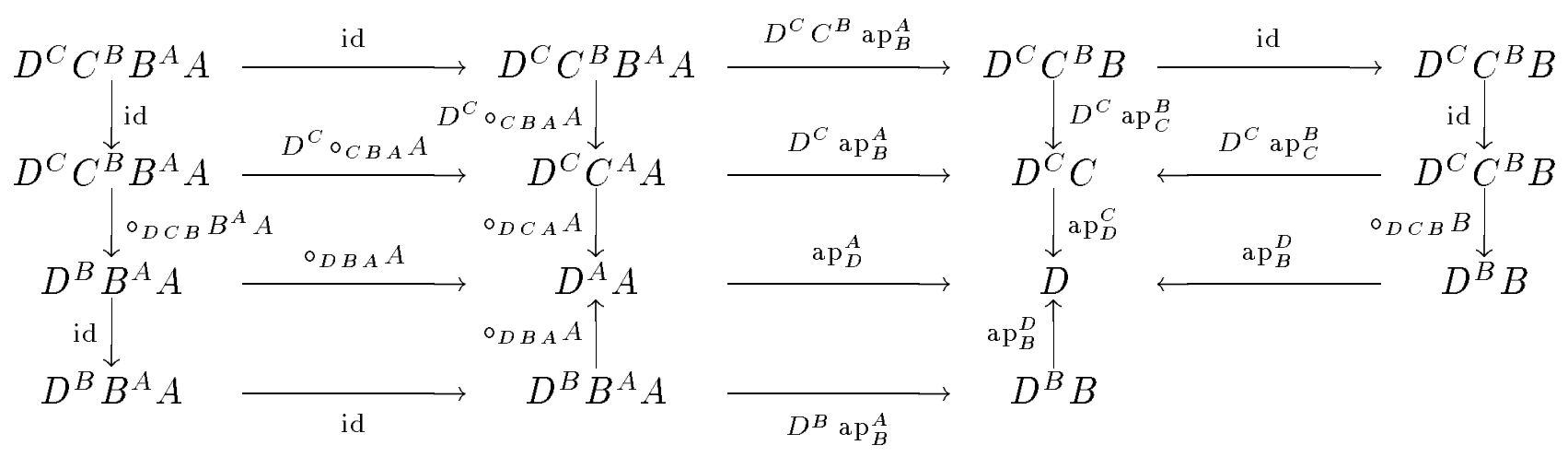


For the purposes of diagram chasing we record the following:

1) The outer contour commutes by the naturality of $\operatorname{ap}_{B}^{A}$.

2) The corner rectangles commute trivially.

3) The upper middle rectangle commutes because it is of the form $D^{C}(*)$.

4) The middle right rectangle, the middle bottom rectangle, and the center rectangle commute because they are of the form $(*)$

Simple diagram chasing now shows that the paths around the left middle rectangle followed by ap ${ }_{D}^{A}$ are equal. This is equation (2).

An object $E$ in a category $\mathcal{C}$ is called terminal if for each object $A$ there is a unique morphism $c_{A E}: A \rightarrow E$. If $E_{1}$ and $E_{2}$ are terminal, then there is a unique isomorphism $c_{E_{1} E_{2}}: E_{1} \rightarrow E_{2}$ with $c_{E_{1} E_{2}}^{-1}=c_{E_{2} E_{1}}$. In this sense one may even speak of "the" terminal object.

Proposition A.6. Suppose that $\mathcal{C}$ is a cartesian closed category with a terminal object $E$. Then

(i) $\mathrm{pr}_{1}: A E \rightarrow A$ and $\mathrm{pr}_{2}: E A \rightarrow A$ are isomorphisms,

(ii) the constant-picker $\kappa_{X}^{E}: X \rightarrow X^{E}$ is an isomorphism.

(iii) If $\mathcal{C}$ has products then there is a natural morphism $\gamma_{B}^{A}: B^{A} \rightarrow B^{\mathcal{C}(E, A)}$ given by the universal property of the product through the following diagram

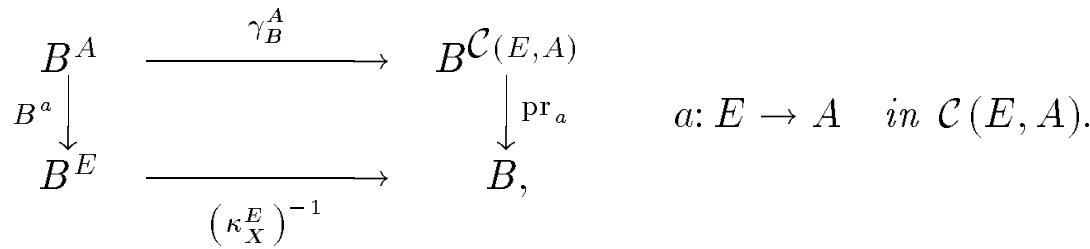

Proof. (i) There is a unique $\sigma_{A}: A \rightarrow A E$ such that $\mathrm{pr}_{1} \circ \sigma_{A}=\mathrm{id} \mathrm{d}_{A}$ and $\mathrm{pr}_{2} \circ \sigma_{A}=$ $(A \rightarrow E)$. Set $\phi=\sigma_{A} \circ \mathrm{pr}_{1}: A E \rightarrow A E$. Then $\operatorname{pr}_{1} \circ \phi=\operatorname{pr}_{1} \circ \sigma_{A} \circ \mathrm{pr}_{1}=\mathrm{pr}_{1}$, and $\mathrm{pr}_{2} \circ \phi: A E \rightarrow E$ is the unique terminal morphism. The identity morphism $\operatorname{id}_{A E}: A E \rightarrow A E$ has the same properties; the uniqueness in the universal property of a product shows that $\phi=\mathrm{id} A E$. We have seen that $\sigma_{A}=\operatorname{pr}_{1}^{-1}$.

Similarly one shows that the projection $E A \rightarrow A$ is an isomorphism. (This also follows from the preceding and the commutativity of the product.)

(ii) With the natural isomorphism $\sigma_{E}=\operatorname{pr}_{1}^{-1}: X \rightarrow X E$ as in (i) above the following commuting diagram shows that $\kappa_{X}^{E}: X \rightarrow X^{E}$ is a coretraction:

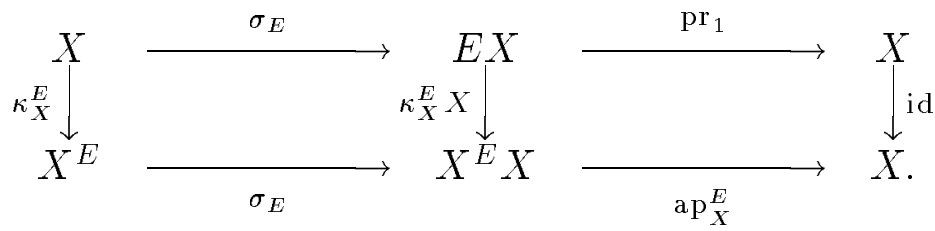

This coretraction is an isomorphism iff its left inverse $\operatorname{ap}_{X}^{E}: X^{E} E \rightarrow X$ is monic. Thus let $U$ be an arbitrary object and $\alpha, \beta: U \rightarrow X^{E} E$ two morphisms with ap $\circ \alpha=$ ap $\circ \beta$. Since $U \cong U E$ by (i) above, we may assume w.l.g. that $\alpha, \beta: U E \rightarrow X^{E} E$. By (i) there are unique morphisms $\alpha^{\prime}, \beta^{\prime}: U \rightarrow X^{E}$ with $\alpha^{\prime} E=\alpha$ and $\beta^{\prime} E=\beta$. The uniqueness in Remark 2 now shows that $\alpha^{\prime}=\beta^{\prime}$ and this means $\alpha=\beta$. Hence $\operatorname{ap}_{X}^{E}$ is a monic and thus Claim (ii) is established. immediate.

By (ii) the constant-picker $\kappa_{X}^{E}$ is an isomorphism. The proof of (iii) is now 
Definition A.7. (i) A morphism $m: A A \rightarrow A$ in a cartesian closed category is called associative if the following diagram commutes.

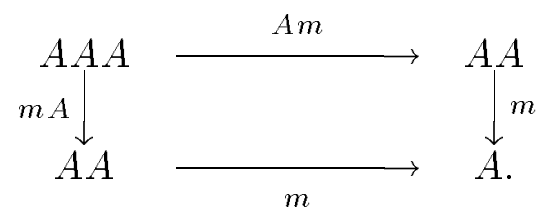

An object $(A, m)$ together with an associative multiplication is called a semigroup in $\mathcal{C}$ or, in short, a $\mathcal{C}$-semigroup.

(ii) If $\mathcal{C}$ has a terminal object $E$, then a morphism $i: E \rightarrow A$ is called an identity of the semigroup $(A, m)$ if the following diagram commutes:

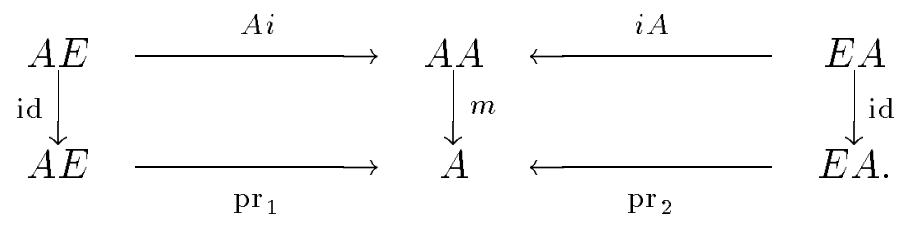

A $\mathcal{C}$-object $(A, m, i)$ with an associative multiplication $m$ and an identity $i$ is called a monoid in $\mathcal{C}$ or, in short, a $\mathcal{C}$-monoid.

(iii) $\mathrm{A}$ morphism $f$ of monoids $\left(A_{1}, m_{1}, i_{1}\right) \rightarrow\left(A_{2}, m_{2}, i_{2}\right)$ is a $\mathcal{C}$-morphism $f: A_{1} \rightarrow A_{2}$ such that

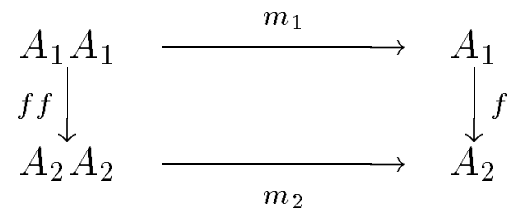

commutes and that $f i_{1}=i_{2}$ holds. We say that $\left(A_{1}, m_{1}, i_{1}\right)$ is a submonoid of $\left(A_{2}, m_{2}, i_{2}\right)$ if there is a monomorphisms $f$ of monoids from the former to the latter.

(iv) A morphism $a: A \times X \rightarrow X$ with a monoid $A$ is called a $\mathcal{C}$-action if the following diagrams commute:
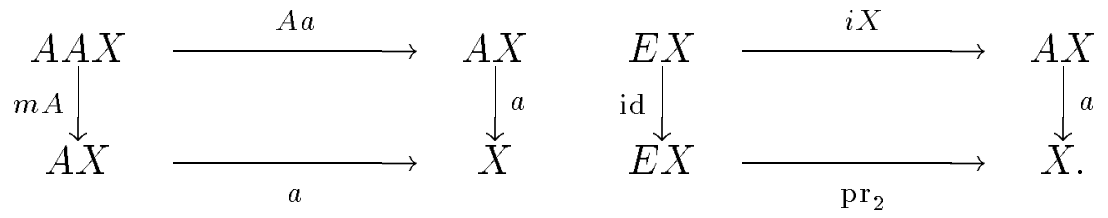

Corollary A.8. In a cartesian closed category $\mathcal{C}$, for each object $A$ in $\mathcal{C}$, the morphism $\circ_{A}=\circ_{A A A}: A^{A} A^{A} \rightarrow A$ makes $A^{A}$ into a semigroup in $\mathcal{C}$.

Note that for the terminal object $E$ the product $E E$ is terminal by A.6(i). Then $\Delta=\Delta_{E}: E \rightarrow E E$ is the unique isomorphism from $E$ to $E E$.

Proposition A.9. Suppose that $\mathcal{C}$ is a cartesian closed category with a terminal object E. Then

(i) The unique morphism $i_{A}: E \rightarrow A^{A}$ defined by the universal property via

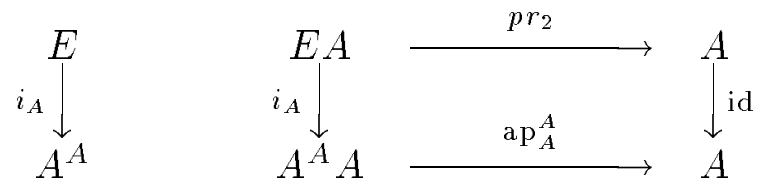


is an identity for the semigroup $\left(A^{A}, \circ_{A}\right)$. I.e., $\left(A^{A}, \circ_{A}, i_{A}\right)$ is a monoid in the category $\mathcal{C}$.

(ii) The monoid structure on $A^{A}$ induces a monoid structure on the set $\mathcal{C}\left(E, A^{A}\right)$. Its multiplication given by

$$
*=\left(\mathcal{C}\left(E, A^{A}\right) \times \mathcal{C}\left(E, A^{A}\right) \longrightarrow \mathcal{C}\left(E, A^{A} A^{A}\right) \stackrel{\mathcal{C}\left(E, \circ_{A}\right)}{\longrightarrow} \mathcal{C}\left(E, A^{A}\right)\right),
$$

$\rho(f, g)=(f g) \circ \Delta$. Its identity is $i_{A}$.

The set $\mathcal{C}(A, A)$ is a monoid w.r.t. ordinary composition o of functions.

(iii) The function $\delta: \mathcal{C}(A, A) \rightarrow \mathcal{C}\left(E, A^{A}\right)$ which associates with a morphism $f: A \rightarrow A$ the unique morphism $\delta(f)=\widetilde{f}: E \rightarrow A^{A}$ characterized by

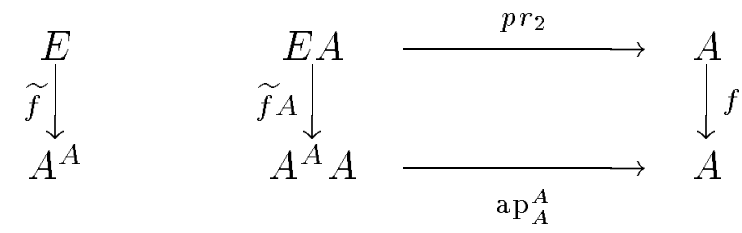

is an isomorphism of monoids.

Proof. (i) We have to show that the following diagram (and an analogous one in which factors are exchanged) commutes:

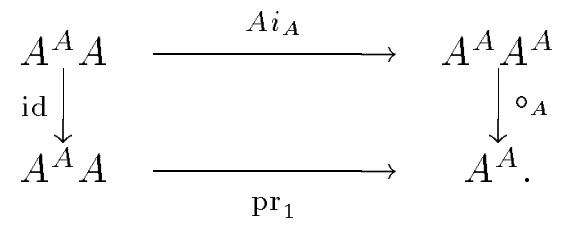

In other words, we set $\alpha=\left(\circ_{A}\right) \circ\left(A^{A} i_{A}\right): A^{A} E \rightarrow A^{A}$ and $\beta=\mathrm{pr}_{1}: A^{A} E \rightarrow A^{A}$, and want to show that $\alpha=\beta$. By Remark 3 it suffices to show that

$$
\operatorname{ap}_{A}^{A} \circ(\alpha A)=\operatorname{ap}_{A}^{A} \circ(\beta A) \text {. }
$$

Now we note from the definition of the product and its associativity that

$$
A^{A} \operatorname{pr}_{2}=\operatorname{pr}_{1} A: A^{A} E A \rightarrow A^{A} A
$$

With (2) equation (1) becomes

$$
\operatorname{ap}_{A}^{A} \circ\left(\left(\circ_{A} A\right) \circ\left(A^{A} i_{A} A\right)\right) A=\operatorname{ap}_{A}^{A} \circ\left(A^{A} \operatorname{pr}_{2}\right) .
$$

We have the commuting of

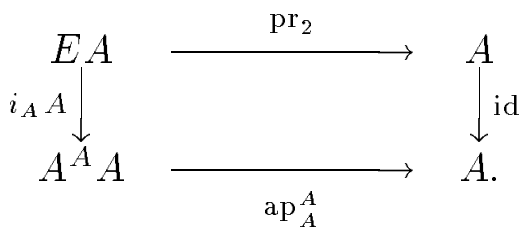

We multiply on the right by $A$ and complete below with the defining diagram for $\circ_{A}$ :

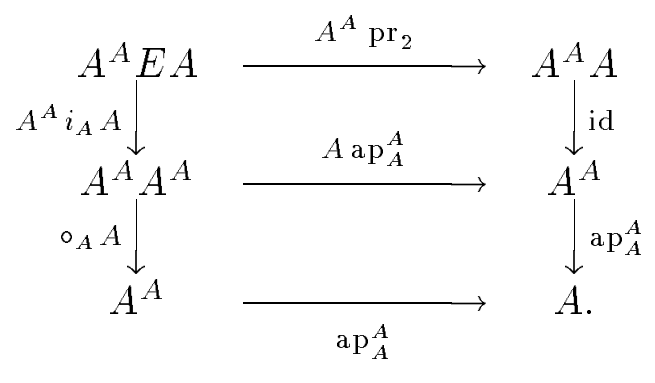


But the commuting of this diagram yields (3), which we had to show.

The commuting of the diagram for the identity on the other side has an analogous proof.

(ii) The functor $X \mapsto \mathcal{C}(E, X): \mathcal{C} \rightarrow \mathcal{S E \mathcal { T }}$ preserves products. Product preserving functors map monoids in the domain category to monoids in the range category. This proves the first assertion.

The second assertion follows directly from the axioms of a category.

(iii) The function $\delta$ is a natural bijection of sets by the adjunction property. The monoid multiplication $*: \mathcal{C}\left(E, A^{A}\right) \times \mathcal{C}\left(E, A^{A}\right) \rightarrow \mathcal{C}\left(E, A^{A}\right)$ is defined as follows: For two given morphisms $f, g: A \rightarrow A$ the morphism $\widetilde{f} * \widetilde{g}: \mathcal{C}\left(E, A^{A}\right) \rightarrow \mathcal{C}\left(E, A^{A}\right)$ is the following composition of maps

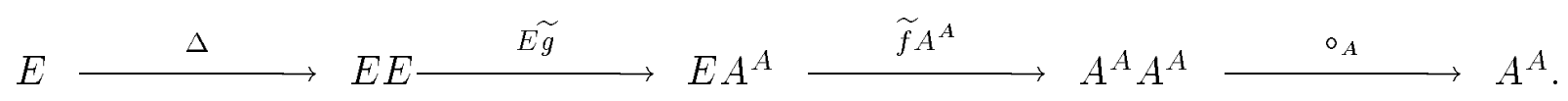

We want to show that $\delta(f \circ g)=\widetilde{f} * \widetilde{g}$. Following Remark 3 we shall show

$$
\operatorname{ap}_{A}^{A} \circ(\delta(f \circ g)) A=\operatorname{ap}_{A}^{A} \circ(\widetilde{f} * \widetilde{g}) A \text {. }
$$

The commutativity of the following diagram will show this claim:

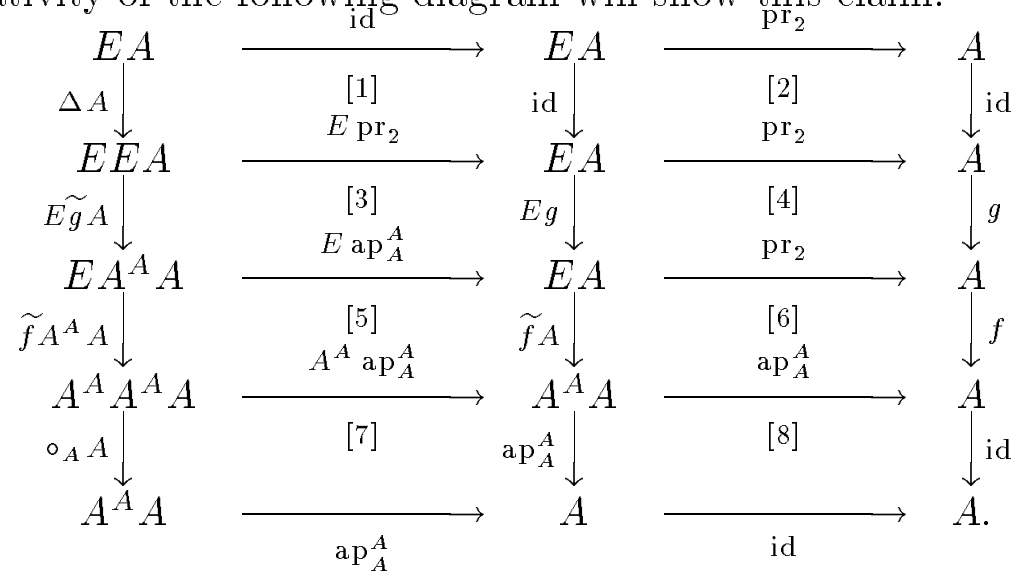

The individual cells commute for the following reasons

[1] $E \stackrel{\Delta}{\rightarrow} E E \stackrel{\mathrm{Pr}_{1}}{\rightarrow} E$ is the identity of $E$.

[2] Trivial.

[3] This cell is of the type $E(\dagger)$ for $g$.

[4] Trivial.

[5] Functoriality of the product.

[6] This is diagram ( $\dagger$ ) for $f$.

[7] Definition of composition in Remark 3.

[8] Trivial.

Thus the diagram is commutative and shows (4). Every isomorphism of semigroups preserves identities and thus is an isomorphism of monoids.

Comparing the diagrams in (ii) (defining $i_{A}$ ) and $(\dagger)$ (defining $f \mapsto \widetilde{f}$ ) we see that $\delta\left(\operatorname{id}_{A}\right)=i_{A}$. Since isomorphisms of semigroups preserve identities we see that $i_{A}$ is the identity of the semigroup $\left.\mathcal{C}\left(E, A^{A}\right), *\right)$

In a category $\mathcal{C}$ with a terminal object $E$ one says that a morphism $f: A \rightarrow B$ is constant if it factors through $E$, i.e., if there is a morphism $b: E \rightarrow B$ such that with the unique $c_{A}: A \rightarrow E$ one has $f=b \circ c_{A}$. The map $b \mapsto b \circ c_{A}: \mathcal{C}(E, B) \rightarrow \mathcal{C}(A, B)$ maps $\mathcal{C}(E, B)$ bijectively onto the set $\mathcal{C}_{\text {const }}(A, B) \subseteq \mathcal{C}(A, B)$ constant morphisms $A \rightarrow B$. 
Remark A.10. The constant-picker $\kappa_{B}^{A}: B \rightarrow B^{A}$ of A.4 induces a bijection $\mathcal{C}(E, B) \rightarrow \mathcal{C}_{\text {const }}(A, B)$ via the function

$$
\gamma=\left(\mathcal{C}(E, B) \stackrel{\mathcal{C}\left(E, \kappa_{B}^{A}\right)}{\longrightarrow} \mathcal{C}\left(E, A^{B}\right) \stackrel{\cong}{\rightrightarrows} \mathcal{C}(E A, B) \stackrel{\mathcal{C}\left(\operatorname{pr}_{1}, B\right)}{\longrightarrow} \mathcal{C}(A, B)\right)
$$

Proof. We consider the diagram
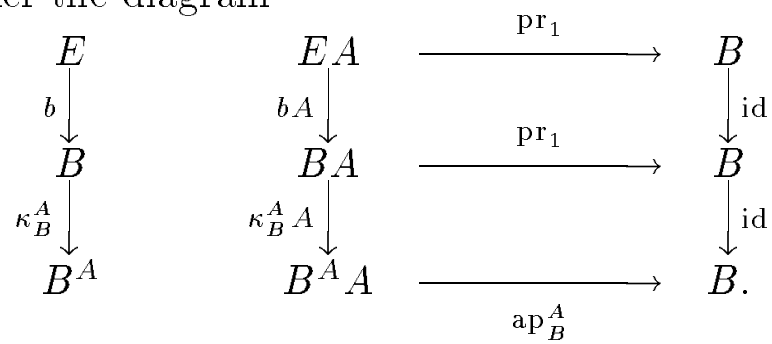

Since $\mathcal{C}(E, \kappa)(b)=\kappa \circ b$, the function $\gamma$ assigns to $b: E \rightarrow B$ bijectively the function $A \stackrel{c_{A}}{\rightarrow} E \stackrel{b}{\rightarrow} B$.

Definition A.11. We call a cartesian closed category concrete or set based if it has products and there is a faithful and product preserving functor $|\cdot|: \mathcal{C} \rightarrow \mathcal{S E} \mathcal{T}$.

If $|\cdot|: \mathcal{C} \rightarrow \mathcal{S E} \mathcal{T}$ is a faithful functor, and if $|E|$ is singleton, then each constant morphism $f$ gives a constant function $|f|:|A| \rightarrow|B|$. We say that all constant maps are morphisms in $\mathcal{C}$ if the image of $\mathcal{C}(A, B)$ in $|B|^{|A|}$ under $|\cdot|$ contains all constant functions.

Remark A.12. (i) If $E$ is a terminal object in a set-based category, then $|E|$ is a one element set $\{*\}$.

(ii) The function $j_{A}: \mathcal{C}(E, A) \rightarrow|A|, j_{A}(f)=|f|(*)$ is injective.

(iii) The following conditions are equivalent:

(1) For all objects $A$ the function $j_{A}: \mathcal{C}(E, A) \rightarrow|A|$ is bijective.

(2) All constant maps are morphisms in $\mathcal{C}$.

Proof. (i) and (ii): $\mathrm{By}$ A.6(i) we know that $\mathrm{pr}_{1}: A E \rightarrow A$ is an isomorphism. Then since $|\cdot|$ preserves products $\operatorname{pr}_{1}:|A| \times|E| \rightarrow|E|$ is bijective. Letting $A=E$ we get $\operatorname{card}|E|=1$. This proves (i), and (ii) follows since the map $\mathcal{C}(E, A) \rightarrow$ $\mathcal{S E T}(|E|,|A|)=|A|^{|E|}$ is injective as $|\cdot|$ is faithful.

(iii) (1) implies (2): Suppose that $c:|A| \rightarrow|B|$ is a constant function. If $b$ is its value, then by (i) there is a unique morphism $f_{b}: E \rightarrow B$ with $b=\left|f_{b}\right|(*)$. Let $c_{A}: A \rightarrow E$ be the unique morphism into the terminal object $A$. Set $f=f_{b} \circ c_{A}$. Then $|f|=(* \mapsto b) \circ(|A| \rightarrow\{*\})$ and this function is $c$.

(2) implies (1): Immediate from the definitions.

Definition A.13. We say that a concrete cartesian closed category $\mathcal{C}$ has function spaces if it has a terminal object and the equivalent conditions of A.12(iii) are satisfied.

In a concrete category with function spaces we have $\mathcal{C}(A, B)=\left|A^{B}\right|$ (according to $A .12)$. The grounding functor $|\cdot| \cong \mathcal{C}(E, \cdot)$ assigns to the constant-picker $\kappa_{B}^{A}: B \rightarrow B^{A}$ the function $x \mapsto \kappa_{x}:|B| \rightarrow|B|^{|A|}$ where $\kappa_{x}$ is the constant function with value $x \in|B|$.

In the sense of Proposition A.9(iii), the monoid $\mathcal{C}(A, A)$ is the underlying monoid of the $\mathcal{C}$-monoid $A^{A}$.

Recall that in any concrete category $\mathcal{C}$ a morphism $f: X \rightarrow Y$ is called injective if $|f|:|X| \rightarrow|Y|$ is injective. Since $|$.$| is faithful, injective morphisms are$ monics. 
Proposition A.14. In a concrete cartesian closed category with function spaces the morphism $\gamma_{B}^{A}: B^{A} \mapsto B^{\mathcal{C}(E, A)}$ of A.6(iii) is injective.

Proof. Since |.|: $\mathcal{C} \rightarrow \mathcal{S E \mathcal { T }}$ preserves products we have

$$
\left|\gamma_{B}^{A}\right|:\left|B^{A}\right| \mapsto|B|^{\mathcal{C}(E, A)}
$$

and $|\gamma|$ is uniquely determined by the conditions that

$$
\operatorname{pr}_{a} \text { o| } \mid=\left(\left|B^{A}\right| \longrightarrow\left|B^{a}\right| \longrightarrow\left|B^{E}\right| \longrightarrow\left|\kappa_{E}^{B}\right|\right)
$$

for all $a: E \rightarrow A$. We may replace $\left|B^{A}\right|$ by $\mathcal{C}(A, B)$ and $\left|B^{E}\right|$ by $\mathcal{C}(E, B)$, accordingly, $\left|B^{a}\right|$ by $\mathcal{C}(a, B)$ and $\left|\kappa_{E}^{B}\right|$ by $j_{B}: \mathcal{C}(E, B) \rightarrow|B|$. Thus take $f_{j} \in$ $\mathcal{C}(A, B), j=1,2$. Then $\operatorname{pr}_{a}\left(|\gamma|\left(f_{j}\right)\right)=j_{B}\left(\mathcal{C}(a, B)\left(f_{j}\right)\right)=j_{B}\left(f_{j} \circ a\right)=f_{j}(a(*))$, and the equality of these two expressions for $j=1,2$ and for all $a$ implies $f_{1}=f_{2}$.

In view of $\mathcal{C}(E, A) \cong|A|$ in a concrete cartesian closed category the morphism $\gamma_{B}^{A}: B^{A} \rightarrow B^{\mathcal{C}(E, A)}$ may be replaced by

$$
\gamma_{B}^{A}: B^{A} \mapsto B^{|A|}
$$

which induces the injective function

$$
\left|\gamma_{B}^{A}\right|:\left|B^{A}\right| \rightarrow\left|B^{|A|}\right|=|B|^{|A|}
$$

\section{References}

[1] Adámek, J., H. Herrlich, and G. Strecker, Abstract and Concrete Categories, Wiley-Interscience, New York etc., 1990, xii+482 pp.

[2] Barendregt, H. P., The Lambda Calculus, North-Holland, Amsterdam etc., 1981, xiv+615pp.

[3] Clifford, A. H. and G. B. Preston, The Algebraic Theory of Semigroups, Volume I, American Mathematical Society, Providence (1961), 224pp.

[4] Gierz, G., K. H. Hofmann, K. Keimel, J. Lawson, M. Mislove and D. S. Scott, A Compendium of Continuous Lattices, Springer-Verlag, Heidelberg, New York (1980), 369pp.

[5] Hofmann, K. H. and P. S. Mostert, Elements of Compact Semigroups, Charles Merrill \& Co., Columbus (1984), 384pp.

[6] Meyer, Albert, What is a model of the lambda calculus?, Information and Control 57 (1982), $87-122$.

[7] Scott, D. S., Continuous lattices, Lecture Notes in Mathematics 274 (1972), Springer-Verlag, Heidelberg, New York, $97-136$. 